\title{
Pricing Vulnerable European Options under Lévy Process with Stochastic Volatility
}

\author{
Chaoqun Ma, Shengjie Yue $(\mathbb{D}$, and Yishuai Ren \\ Business School of Hunan University, Changsha 410082, China \\ Correspondence should be addressed to Shengjie Yue; yueshengjie18@163.com
}

Received 5 July 2018; Accepted 30 September 2018; Published 23 October 2018

Academic Editor: Daniel Sevcovic

Copyright (c) 2018 Chaoqun Ma et al. This is an open access article distributed under the Creative Commons Attribution License, which permits unrestricted use, distribution, and reproduction in any medium, provided the original work is properly cited.

\begin{abstract}
This paper considers the pricing issue of vulnerable European option when the dynamics of the underlying asset value and counterparty's asset value follow two correlated exponential Lévy processes with stochastic volatility, and the stochastic volatility is divided into the long-term and short-term volatility. A mean-reverting process is introduced to describe the common long-term volatility risk in underlying asset price and counterparty's asset value. The short-term fluctuation of stochastic volatility is governed by a mean-reverting process. Based on the proposed model, the joint moment generating function of underlying log-asset price and counterparty's log-asset value is explicitly derived. We derive a closed-form solution for the vulnerable European option price by using the Fourier inversion formula for distribution functions. Finally, numerical simulations are provided to illustrate the effects of stochastic volatility, jump risk, and counterparty credit risk on the vulnerable option price.
\end{abstract}

\section{Introduction}

In general, options traded in the over-the-counter (OTC) market are associated with counterparty credit risk. Since the global financial crisis of 2007-2008, regulators and investors have widely recognized the effect of counterparty credit risk on OTC options (see, e.g., Crépey [1], Sayah [2], and Breton and Marzouk [3]). Unlike the options traded in regulated exchanges, there is no organized exchange to ensure that option writers will fulfill the contractual obligations in the OTC markets. Once the option writer defaults, the option holder will get only a fraction of necessary payments required by the contract. The holders of OTC options are likely to suffer a large loss if they ignore the counterparty credit risk. Thus, the participants in the OTC markets need to take into account counterparty credit risk when pricing OTC options. Those options with counterparty credit risk are referred to as vulnerable options.

The valuation of vulnerable options has gained substantial attention in the finance literature. For modeling credit risk, the structural approach has been used to study vulnerable options by a lot of scholars, in which default events happen when the firm's asset value is lower than a specific boundary.
Based on this approach, the vulnerable European option pricing model is first presented by Johnson and Stulz [4]. Klein [5] extends the work of Johnson and Stulz [4] by assuming option writer to have other liabilities, in which the prices of underlying asset and the asset of counterparty are correlated. Subsequently, many extensions of the results in Klein [5] have been considered. For example, Hui et al. [6] and Hui et al. [7] discuss the pricing of vulnerable European option with stochastic default barrier. In contrast to constant interest rate in Klein [5], Klein and Inglis [8] incorporate stochastic interest rate into the vulnerable option pricing model. Liao and Huang [9] consider the possible counterparty default prior to the maturity of option; they derive explicit formulas for vulnerable options with stochastic interest rates. Taking jump into account, Tian et al. [10] investigate the vulnerable European option pricing where the dynamics of asset prices are governed by two correlated jump-diffusion processes. More broadly, Niu and Wang [11] discuss the pricing of vulnerable European option under regime switching setting.

However, the above mentioned literature generally assume that the volatilities of the underlying asset and counterparty's asset are constant, which is contrary to the actual situation, because it cannot explain many well-known 
empirical evidences such as the volatility clustering and the volatility smile. It is obvious that models with stochastic volatility will be more realistic. Some scholars have suggested alternative models for the price of vulnerable option. Lee et al. [12] derive the explicit expression for the vulnerable option price where the price processes of underlying asset and counterparty's asset satisfy the Heston stochastic volatility model. Wang et al. [13] extend the model of Lee et al. [12] and assume that the price processes of underlying asset and counterparty's asset follow two correlated two-factor stochastic volatility diffusion processes; they obtain a pricing formula of vulnerable option in a special case. However, Lee et al. [12] and Wang et al. [13] do not consider the effects of jump risk of underlying asset and counterparty's asset on vulnerable option price. A lot of empirical evidences show that the arrival of new information and sudden disastrous events may lead to discontinuous changes in asset prices and the jump components of models are understood as the effects of market shocks on the asset prices. Therefore, many researchers have proposed pricing models that incorporate both stochastic volatility and jumps components. For example, Bates [14] studies pricing problem of option where the dynamics of underlying asset follows a jump-diffusion process with stochastic volatility, in which the jump components of asset price follow the compound Poisson process. However, Carr and $\mathrm{Wu}$ [15] and Huang and $\mathrm{Wu}$ [16] find the fact that the compound Poisson process cannot describe the many small jumps of financial assets. Moreover, many researches have considered more general jump structures. A more general process is Lévy process, which allows the jump components to have infinite activity and admits nearly an arbitrary distribution. Li et al. [17] apply Markov chain Monte Carlo (MCMC) methods to study infinite-activity Lévy processes with stochastic volatility; their empirical results show that infinite-activity jumps are essential for modeling the S\&P 500 index returns. Subsequently, Zaevski et al. [18] derive the general formula for a European call option under stochastic volatility and tempered stable Lévy jumps. Liang and $\mathrm{Li}$ [19] study pricing of the European option under Normal Tempered Stable process with stochastic volatility. Li et al. [20] discuss the equity premium and option pricing under the general equilibrium under Lévy process and stochastic volatility.

Along with the above research lines, based on the structural approach for default, we study the valuation of vulnerable European option when the price processes of the underlying asset and counterparty's asset follow two correlated exponential Lévy processes with stochastic volatility. The volatilities of underlying asset and counterparty's asset are both decomposed into long-term and short-term volatility. The short-term variance of asset price follows a meanreverting process. A mean-reverting process is introduced to allow for the common long-term volatility risk in the underlying asset price and counterparty's asset value, which differs from assumption that long-term volatility is constant in Wang et al. [13]. This assumption is consistent with the models in Christoffersen et al. [21] and Wong and Zhao [22]. The market is fluctuating in reality, and the market factor affects the longterm volatilities of underlying asset price and counterparty's asset value. Thus, the long-term volatility of asset price is also changing over time. Based on the proposed model, the joint moment generating function of underlying log-asset price and counter's log-asset value is derived. Moreover, we obtain an explicit formula for vulnerable European call option price by applying the Fourier inversion transform. Finally, sensitivity analysis and numerical simulation are provided for our results. By the numerical analysis, we discover that (i) the long-run mean of common long-term variance of underlying asset and counterparty's asset exerts positive effect on the vulnerable option price; (ii) vulnerable option price is an increasing function with respect to the long-run mean of short-term variance of underlying asset price; (iii) a higher long-run mean of short-term variance of counterparty's asset value induces a lower vulnerable option price; (iv) when the jump components of underlying asset price and counterparty' asset value follow the CGMY model, the aggregate activity rate of jumps of underlying asset price has positive effect on the value of vulnerable option price. The higher aggregate activity rate of jumps of counterparty's asset value, the lower vulnerable option price.

Our contributions to the literature are summarized below: First, we extend the model of Wang et al. [13] by considering a more general financial market. In particular, compared to the constant long-term volatilities of underlying asset price and counterparty's asset value in Wang et al. [13], we assume that the common long-term volatility risk in the underlying asset price and counterparty's asset value is governed by a meanreverting process. Besides, we simultaneously incorporate infinite-activity Lévy jumps and stochastic volatility into the dynamics of underlying asset and counterparty's asset, which differs from the jump-free model in Wang [13]. Second, we derive an explicit expression for vulnerable option price by the Fourier inversion formula for distribution functions, which is different from the methods in Lee et al. [12] and Wang et al. [13].

The rest of this paper is organized as follows. Section 2 introduces the basic model setup and formulates the pricing problem of vulnerable European call option problem. In Section 3, the joint characteristic function of the underlying log-asset price and counterparty's log-asset value is derived. By applying Fourier inversion transform, we derive an analytic solution for vulnerable European option price. Section 4 demonstrates our results with numerical examples, and Section 5 concludes this paper.

\section{Basic Setting of the Model}

In this section, we describe theoretical framework for valuing of vulnerable European call option. The underlying asset price and counterparty's asset value follow exponential Lévy processes with stochastic volatility. The volatility risk is divided into short-term risk and long-term risk. We incorporate a mean-reverting process to describe the common longterm volatility risk in underlying asset price and counterparty's asset value. The short-term fluctuation of stochastic volatility also follows a mean-reverting process.

Time is indexed by $t \in[0, T]$, where $\mathrm{T}$ is a positive finite constant representing the time horizon. To model uncertainties, suppose that we have a given complete probability space 
with filtration $\left(\Omega, \mathscr{F},\left\{\mathscr{F}_{t}\right\}_{0 \leq t \leq T}, Q\right)$, and the filtration $\left\{\mathscr{F}_{t}\right\}$ satisfies the usual condition (increase, right-continuous and augmented). It represents the flow of information available to the investor. $Q$ is a risk-neutral probability. Suppose $W_{1}^{S}$, $W_{2}^{S}, W_{j}^{Z}(j=1,2,3), W_{1}^{V}$, and $W_{3}^{V}$ are all standard Brownian motions which are $\mathscr{F}_{t}$-adapted. Moreover, $\operatorname{cov}\left(d W_{1}^{S}(t)\right.$, $\left.d W_{1}^{Z}(t)\right)=\rho_{1 s} d t, \operatorname{cov}\left(d W_{2}^{S}(t), d W_{2}^{Z}(t)\right)=\rho_{2 s} d t, \operatorname{cov}\left(d W_{1}^{V}(t)\right.$, $\left.d W_{1}^{Z}(t)\right)=\rho_{1 v} d t, \operatorname{cov}\left(d W_{3}^{V}(t), d W_{3}^{Z}(t)\right)=\rho_{3 v} d t, \operatorname{cov}\left(d W_{1}^{S}(t)\right.$, $\left.d W_{1}^{V}(t)\right)=\rho_{s v} d t$, and any other Brownian motions are pairwise independent. Under a risk neutral measure $Q$, the underlying asset price $S(t)$ is modeled as the exponential of the lévy process with stochastic volatility:

$$
\begin{aligned}
\frac{d S(t)}{S(t-)}= & r d t+\eta_{1} \sqrt{Z_{1}(t)} d W_{1}^{S}(t)+\sqrt{Z_{2}(t)} d W_{2}^{S}(t) \\
& +\int_{R}\left(e^{y}-1\right) \widetilde{N}_{y}(d t, d y), \\
d Z_{1}(t)= & \kappa_{1}\left(\theta_{1}-Z_{1}(t)\right) d t+\sigma_{1} \sqrt{Z_{1}(t)} d W_{1}^{Z}(t), \\
d Z_{2}(t)= & \kappa_{2}\left(\theta_{2}-Z_{2}(t)\right) d t+\sigma_{2} \sqrt{Z_{2}(t)} d W_{2}^{Z}(t),
\end{aligned}
$$

where $S(t-)$ stands for the value of $S(t)$ before a possible jump occurs, $y \in R=\mathbb{R} \backslash\{0\} ; r$ is the interest rate, and the parameters $\kappa_{j}, \theta_{j}$, and $\sigma_{j}(j=1,2)$ are the mean-reverting rate, the long-term mean; and volatility of the variance process $Z_{j}(t)$, respectively. We require $2 \kappa_{j} \theta_{j} \geq \sigma_{j}^{2}$ to ensure that the process $Z_{j}(t)$ remains strictly positive. $\eta_{1}^{2} Z_{1}(t)$ represents the long-term variance of underlying asset price, which reflect the effect of corporate performance and market states on the underlying asset price. Here we assume that $Z_{1}(t)$ follow a mean-reverting process, which represents the common systematic risk caused by market factors. $Z_{2}(t)$ is shortterm variance of underlying asset value, which depends on investors' trading and reflects company's idiosyncratic risk. In addition, $\widetilde{N}_{y}(d t, d y)=N_{y}(d t, d y)-v_{y}(d y) d t$ is a compensated jump measure, where $N_{y}(d t, d y)$ is the jump measure and Lévykernel (or density) $v_{y}(d y)$ satisfies $\int_{R} \min \left(1, y^{2}\right) v_{y}(d y)<$ $\infty$; the underlying asset price in (1) contains two orthogonal martingales: two purely continuous martingale and a purely discontinuous (jump) martingale.

Next, let $V(t)$ denote the market value of counterparty's asset, and the dynamic of $V(t)$ is given by the SDE under risk neutral measure $Q$,

$$
\begin{aligned}
\frac{d V(t)}{V(t-)}= & r d t+\eta_{2} \sqrt{Z_{1}(t)} d W_{1}^{V}(t)+\sqrt{Z_{3}(t)} d W_{3}^{V}(t) \\
& +\int_{R}\left(e^{\xi}-1\right) \widetilde{N}_{\xi}(d t, d \xi) \\
d Z_{3}(t)= & \kappa_{3}\left(\theta_{3}-Z_{3}(t)\right) d t+\sigma_{3} \sqrt{Z_{3}(t)} d W_{3}^{Z}(t),
\end{aligned}
$$

where $V(t-)$ stands for the value of $V(t)$ before a possible jump occurs, $\xi \in R=\mathbb{R} \backslash\{0\} ; \eta_{2}^{2} Z_{1}(t)$ represents the long-term variance of counterparty's asset value, and $Z_{1}(t)$ describes the systematic risk caused by market shocks. $Z_{3}(t)$ is short-term variance of counterparty's asset price, which depends on the investor's trading activities. The parameters $\kappa_{3}, \theta_{3}$, and $\sigma_{3}$ are the mean-reverting rate, the long-term mean, and volatility of the variance process $Z_{3}(t)$, respectively. We require $2 \kappa_{3} \theta_{3} \geq \sigma_{3}^{2}$ to ensure that the process $Z_{3}(t)$ remains strictly positive. In addition, $\widetilde{N}_{\xi}(d t, d \xi)=$ $N_{\xi}(d t, d \xi)-v_{\xi}(d \xi) d t$ is a compensated jump measure, where $N_{\xi}(d t, d \xi)$ is the jump measure and Lévy kernel (or density) $\nu_{\xi}(d \xi)$ satisfies $\int_{R} \min \left(1, \xi^{2}\right) \nu_{\xi}(d \xi)<\infty$.

In the proposed framework, we consider stochastic volatility and jumps in the dynamics of both the underlying asset and the counterparty's asset, and the long-term stochastic volatility and the short-term stochastic volatility are not constants. The underlying asset price and the counterparty's asset value are correlated, and the correlation coefficient is $\rho_{S V}$. Furthermore, correlation between stochastic volatility and asset prices is also considered in the proposed model. The short-term stochastic volatilities of the underlying asset and the counterparty reflect company's idiosyncratic risk while the long-term stochastic volatilities of the underlying asset and the counterparty describe systematic risk caused by common market shocks. In addition, we also focus on the discontinuous changes in the underlying asset and counterparty's asset; the jump components in (1) and (4) reflect the abnormal vibrations in the asset price due to the arrival of important new information and sudden disastrous events.

\section{Vulnerable European Call Option Pricing}

In this section, we derive the joint moment generating function of the underlying log-asset price and counterparty' logasset value. In this framework, by using the Fourier inversion transform, we obtain the explicit expression for vulnerable European option price.

Let $T$ denote the option maturity; for vulnerable European call options, the boundary condition is determined by a payoff function depending upon the financial distress circumstance at the time $t=T$. As in Klein (1996) and its subsequent results, then the payoff of a vulnerable European call option is given by

$$
\begin{aligned}
C_{T}= & (S(T)-K)^{+} \mathbf{1}_{\left\{V(T) \geq D^{*}\right\}} \\
& +\frac{(1-\gamma) V(T)(S(T)-K)^{+}}{D} \mathbf{1}_{\left\{V(T)<D^{*}\right\}},
\end{aligned}
$$

where $K$ is the strike price of the option. $\gamma$ is the deadweight cost related with the bankruptcy or reorganization process of the firm expressed as a percentage of the counterparty' asset value. $D$ is the total amount of claims of the counterparty, and $D^{*}$ is an exogenous default barrier. $D^{*}$ may be less than $D$ due to the possibility of a counterparty continuing its operation. A credit loss happens if the counterparty's asset value $V(T)$ is less than the amount $D^{*}$. If $V(T)$ is higher than or equal to the default barrier $D^{*}$, the claim is paid out in full. Otherwise, default event occurs and only the proportion $(1-\gamma) V(T) / D$ of the nominal claim at $T$ is paid out by the option writer. 
By the standard risk-neutral arguments, under a riskneutral measure $Q$, the price of a vulnerable European call option at time 0 is given as

$$
\begin{gathered}
C(S, V, t, T)=E^{Q}\left[e ^ { - r ( T - t ) } \left((S(T)-K)^{+} \mathbf{1}_{\left\{V(T) \geq D^{*}\right\}}\right.\right. \\
\left.\left.+\frac{(1-\gamma) V(T)(S(T)-K)^{+}}{D} \mathbf{1}_{\left\{V(T)<D^{*}\right\}}\right) \mid \mathscr{F}_{t}\right],
\end{gathered}
$$

where $E^{Q}$ denotes risk neutral expectations. $e^{-r(T-t)}$ denotes the stochastic discount factor. Expression (7) shows that the value of a vulnerable European call option contains two parts which are conditional on whether $V(T)$ is higher or less than the default barrier $D^{*}$ under risk-neutral measure $Q$. If $V(T)$ is higher than $D^{*}$, the option writer can afford to pay all the claim. If $V(T)$ is lower than $D^{*}$, only a proportion of the nominal claim is paid.

In the following, we derive the explicit expression for the value of vulnerable option price by using Fourier inversion formula for the probability distribution functions. In the process of calculation, we need to use the joint moment generating function of $\ln S(T)$ and $\ln V(T)$. Thus, it is necessary for us to give this definition of the joint moment generating function. Given the dynamics of the underlying asset price and the counterparty's asset value under the probability measure $Q$, it is possible to obtain the joint moment generating function of $\ln S(T)$ and $\ln V(T)$. Let $\ln S(t)=x_{1}$, $\ln V(t)=x_{2}$, and the joint moment generating function of $\ln S(T)$ and $\ln V(T)$ under probability measure $Q$ is defined as

$$
f\left(x_{1}, x_{2}, \tau ; \varphi_{1}, \varphi_{2}\right)=E^{\mathrm{Q}}\left[e^{\varphi_{1} \ln S(T)+\varphi_{2} \ln V(T)} \mid \mathscr{F}_{t}\right],
$$

where $T \geq t, \tau=T-t$. Then, the following lemma holds.

Lemma 1. Suppose that underlying asset price and counterpart's asset value follow the dynamics in (1) and (4), respectively. Let $\ln S(t)=x_{1}, \ln V(t)=x_{2}, Z_{1}(t)=z_{1}, Z_{2}(t)=z_{2}, Z_{3}(t)=$ $z_{3}$, and the joint moment generating function of $\ln S(T)$ and $\ln V(T)$ in (8) is given by

$$
f\left(x_{1}, x_{2}, \tau ; \varphi_{1}, \varphi_{2}\right)=e^{\varphi_{1} x_{1}+\varphi_{2} x_{2}+A(\tau) z_{1}+B(\tau) z_{2}+D(\tau) z_{3}+E(\tau)},
$$

where

$$
\begin{aligned}
& A(\tau)=-\frac{a_{1}\left(1-e^{-\Delta_{1} \tau}\right)}{2 \Delta_{1}-\left(\Delta_{1}+b_{1}\right)\left(1-e^{-\Delta_{1} \tau}\right)}, \\
& B(\tau)=-\frac{a_{2}\left(1-e^{-\Delta_{2} \tau}\right)}{2 \Delta_{2}-\left(\Delta_{2}+b_{2}\right)\left(1-e^{-\Delta_{2} \tau}\right)}, \\
& D(\tau)=-\frac{a_{3}\left(1-e^{-\Delta_{3} \tau}\right)}{2 \Delta_{3}-\left(\Delta_{3}+b_{3}\right)\left(1-e^{-\Delta_{3} \tau}\right)}, \\
& E(\tau)=\left(r\left(\varphi_{1}+\varphi_{2}\right)\right. \\
& \quad+\int_{R}\left[e^{\varphi_{1} y}-1-\varphi_{1}\left(e^{y}-1\right)\right] v_{y} d(y)
\end{aligned}
$$

$$
\begin{aligned}
& \left.+\int_{R}\left[e^{\varphi_{2} \xi}-1-\varphi_{2}\left(e^{\xi}-1\right)\right] \nu_{\xi} d(\xi)\right) \tau \\
& -\frac{\kappa_{1} \theta_{1}}{\sigma_{1}^{2}}\left(\left(\Delta_{1}+b_{1}\right) \tau\right. \\
& \left.+2 \ln \left[1-\frac{\Delta_{1}+b_{1}}{2 \Delta_{1}}\left(1-e^{-\Delta_{1} \tau}\right)\right]\right) \\
& -\frac{\kappa_{2} \theta_{2}}{\sigma_{2}^{2}}\left(\left(\Delta_{2}+b_{2}\right) \tau\right)+2 \\
& \cdot \ln \left[1-\frac{\Delta_{2}+b_{2}}{2 \Delta_{1}}\left(1-e^{-\Delta_{2} \tau}\right)\right] \\
& -\frac{\kappa_{3} \theta_{3}}{\sigma_{3}^{2}}\left(\left(\Delta_{3}+b_{3}\right) \tau\right. \\
& \left.+2 \ln \left[1-\frac{\Delta_{3}+b_{3}}{2 \Delta_{3}}\left(1-e^{-\Delta_{3} \tau}\right)\right]\right),
\end{aligned}
$$

where

$$
\begin{aligned}
\Delta_{1} & =\sqrt{b_{1}^{2}+\sigma_{1}^{2} a_{1}}, \\
a_{1} & =\left(\eta_{1}^{2} \varphi_{1}+\eta_{2}^{2} \varphi_{2}-\eta_{1}^{2} \varphi_{1}^{2}-\eta_{2}^{2} \varphi_{2}^{2}-2 \eta_{1} \eta_{2} \rho_{s v} \varphi_{1} \varphi_{2}\right) \\
b_{1} & =\left(\eta_{1} \rho_{1 s} \sigma_{1} \varphi_{1}+\eta_{2} \rho_{1 v} \sigma_{1} \varphi_{2}-\kappa_{1}\right), \\
\Delta_{2} & =\sqrt{b_{2}^{2}+\sigma_{2}^{2} a_{2}} \\
a_{2} & =\left(\varphi_{1}-\varphi_{1}^{2}\right) \\
b_{2} & =\left(\varphi_{1} \sigma_{2} \rho_{2 s}-\kappa_{2}\right) \\
\Delta_{3} & =\sqrt{b_{3}^{2}+\sigma_{3}^{2} a_{3}}, \\
a_{3} & =\left(\varphi_{2}-\varphi_{2}^{2}\right) \\
b_{3} & =\left(\varphi_{2} \sigma_{3} \rho_{3 v}-\kappa_{3}\right) .
\end{aligned}
$$

Proof. See Appendix A.

Now, we turn to derive the pricing formula for vulnerable option pricing. We need to rewrite pricing formula (7) of vulnerable European call option as follows:

$$
\begin{gathered}
C(S, V, t, T)=E^{Q}\left[e ^ { - r ( T - t ) } \left((S(T)-K)^{+} \mathbf{1}_{\left\{V(T) \geq D^{*}\right\}}\right.\right. \\
\left.\left.+\frac{(1-\gamma) V(T)(S(T)-K)^{+}}{D} \mathbf{1}_{\left\{V(T)<D^{*}\right\}}\right) \mid \mathscr{F}_{t}\right] \\
=e^{-r(T-t)}\left(C_{1}(t, T)-K C_{2}(t, T)\right)+e^{-r(T-t)} \\
. \frac{(1-\gamma)}{D}\left(C_{3}(t, T)-K C_{4}(t, T)\right),
\end{gathered}
$$

where $C_{1}(t, T)-C_{4}(t, T)$ are given by 


$$
\begin{aligned}
& C_{1}(t, T)=E^{Q}\left[S(T) \mathbf{1}_{\left\{\ln S(T) \geq \ln K, \ln V(T) \geq \ln D^{*}\right\}} \mid \mathscr{F}_{t}\right], \\
& C_{2}(t, T)=E^{Q}\left[\mathbf{1}_{\left\{\ln S(T) \geq \ln K, \ln V(T) \geq \ln D^{*}\right\}} \mid \mathscr{F}_{t}\right], \\
& C_{3}(t, T) \\
& \quad=E^{Q}\left[S(T) V(T) \mathbf{1}_{\left\{\ln S(T) \geq \ln K, \ln V(T)<\ln D^{*}\right\}} \mid \mathscr{F}_{t}\right], \\
& C_{4}(t, T)=E^{Q}\left[V(T) \mathbf{1}_{\left\{\ln S(T) \geq \ln K, \ln V(T)<\ln D^{*}\right\}} \mid \mathscr{F}_{t}\right] .
\end{aligned}
$$

In the following, from the standard probability theory, we can obtain the probability distribution function by using the joint moment generating function. In Theorem 2, we can derive the explicit expression of vulnerable European option by applying Fourier inversion transform.

Theorem 2. The price of vulnerable option pricing at time $t$ is formally given by

$$
\begin{aligned}
& C(t, T)=e^{-r(T-t)}\left(\Pi_{1}(t, T)+\frac{1}{4} f\left(x_{1}, x_{2}, \tau, 1,0\right)\right. \\
& \left.-K \Pi_{2}(t, T)\right)+e^{-r(T-t)} \frac{(1-\gamma)}{D}\left(\Pi_{3}(t, T)\right. \\
& \quad+\frac{1}{4} f\left(x_{1}, x_{2}, \tau, 1,1\right)-K \Pi_{4}(t, T) \\
& \left.-\frac{1}{4} K f\left(x_{1}, x_{2}, \tau, 0,1\right)\right),
\end{aligned}
$$

where $f\left(x_{1}, x_{2}, \tau ; \varphi_{1}, \varphi_{2}\right)$ is the moment generating function in (8), and $\Pi_{1}(t, T), \Pi_{2}(t, T), \Pi_{3}(t, T)$, and $\Pi_{4}(t, T)$ are given in (B.11), (B.12), (B.17), and (B.22), respectively.

Proof. See Appendix B.

\section{Numerical Analysis}

In this section, we present numerical results of the vulnerable European call option price under Lévy process with stochastic volatility. Numerical examples are presented to investigate the effects of the parameters on the vulnerable European call option. The pricing models of vulnerable option in Klein [5] and Wang et al. [13] and pricing models of nonvulnerable option in Sun [23] and Li et. al [20] are chosen as reference models. Our choice of the four reference models is made to study the effects of stochastic volatility, jump risks, and credit risk on vulnerable option prices. In the following numerical analysis, we change one of the parameter values to illustrate its effect on the price with other parameters taking on the values in Tables 1-3. Our model allows a general distribution for jump components of underlying asset price and counterparty's asset value and thus can be easily deduced to the simple cases such that the jump components follow compound Poisson process in Merton [24] and Kou [25], the CGMY model of Carr et al. [26], etc. By assuming different types of Lévy kernel, our model has different forms. In the following, we will first study a simple case with jump components following compound Poisson processes and then a more general case with the jump components following a CGMY model.
In the numerical analysis, we use the function quadgk available in MATLAB to deal with the single integrals for the Fourier inversion transform. The double integrals for the Fourier inversion transform are computed by using the function integral2 available in MATLAB.

4.1. The Merton Model. When jump components of underlying asset and counterparty's asset follow compound Poisson processes, the jump magnitude of asset price is assumed to be drawn from a log-normal distribution. Furthermore, the Lévy kernels are given by

$$
\begin{aligned}
& \nu_{y}(d y)=\lambda_{1} \frac{1}{\sqrt{2 \pi \delta_{1}^{2}}} \exp \left(-\frac{\left(y-\mu_{1}\right)^{2}}{2 \delta_{1}^{2}}\right) d y \\
& \nu_{\xi}(d \xi)=\lambda_{2} \frac{1}{\sqrt{2 \pi \delta_{2}^{2}}} \exp \left(-\frac{\left(\xi-\mu_{2}\right)^{2}}{2 \delta_{2}^{2}}\right) d \xi
\end{aligned}
$$

where $\lambda_{j}(j=1,2)$ is the jump intensity of the asset process, $\mu_{j}$ is the mean of jump size, and $\delta_{j}^{2}$ is the variance of jump size. Following the Merton [24] and Sun [23], the two integrals in (13) are given by

$$
\begin{aligned}
& \int_{R}\left(e^{\varphi_{1} y}-1\right) \nu_{y}(d y)=\lambda_{1}\left(e^{\varphi_{1} \mu_{1}+(1 / 2) \varphi_{1}^{2} \delta_{1}^{2}}-1\right) \\
& \int_{R}\left(e^{\varphi_{2} \xi}-1\right) \nu_{\xi}(d \xi)=\lambda_{2}\left(e^{\varphi_{2} \mu_{2}+(1 / 2) \varphi_{2}^{2} \delta_{2}^{2}}-1\right) .
\end{aligned}
$$

In Table 2, we will give the specimen values for the model parameters of assets' jump components. Figures 1-5 illustrate the effects of basic parameters on vulnerable option prices where the parameters include the default barrier, long-run mean of stochastic volatility, and jump intensity. Denote prices of nonvulnerable European option in Sun [23] by $C_{N E}$, and let $C_{\mathrm{OU}}$ be values of vulnerable option in proposed model. We denote the values of vulnerable option in Wang et al. [13] and Klein [5] prices by $C_{S V}$ and $C_{K L}$, respectively.

Figure 1 illustrates that the vulnerable option price is a decreasing function of default barrier $D^{*}$. When the default barrier is relatively small, the value of vulnerable European option decreases slowly with $D^{*}$; while the default barrier is close to the initial value of counterparty's asset, the vulnerable option price decreases more dramatically with $D^{*}$. Large default barrier $D^{*}$ will increase the default probability; the counterparty credit risk has a more significant impact on the vulnerable option price if the default barrier increases. In addition, the value in proposed model is higher than those of models proposed by Klein [5] and Wang et al. [13]. The phenomenon is consistent with the results presented in existing literature, because the jump parts of underlying asset increase the chance that the underlying asset price will become very low or very high at maturity; the option holder will gain benefit from the increase of underlying asset price and suffers limited downside risk when underlying asset price decreases. Therefore jump risk enhances option value. Moreover, the value of nonvulnerable option price is higher than those of vulnerable European options. Once the credit 
TABLE 1: parameter Values in the Base Case.

\begin{tabular}{lccc}
\hline Parameter & Value & Parameter & Value \\
\hline Time to maturity & $\mathrm{T}=1$ & Mean-reverting rate of volatility & $k_{3}=2$ \\
Time & $\mathrm{t}=0$ & Long-term mean of volatility & $\theta_{3}=0.05$ \\
Initial stock price & $\mathrm{S}(0)=10$ & Volatility of volatility & $\sigma_{3}=0.4$ \\
Initial volatility value & $Z_{1}(0)=0.05$ & Volatility of the asset & $\eta_{1}=1$ \\
Mean-reverting rate of volatility & $k_{1}=1$ & Volatility of the asset & $\eta_{2}=0.5$ \\
Long-term mean of volatility & $\theta_{1}=0.05$ & Outstanding claims & $D=30$ \\
Volatility of volatility & $\sigma_{1}=0.3$ & Default barrier & $D^{*}=30$ \\
Initial volatility value & $Z_{2}(0)=0.06$ & Deadweight cost & $\gamma=0.4$ \\
Mean-reverting rate of volatility & $k_{2}=2$ & Strike price & $\mathrm{K}=10$ \\
Long-term mean of volatility & $\theta_{2}=0.06$ & Initial asset value & $V(0)=30$ \\
Volatility of volatility & $\sigma_{2}=0.5$ & Correlation coefficient & $\rho_{1 s}=-0.5, \rho_{2 s}=-0.5$ \\
Initial volatility value & $Z_{3}(0)=0.05$ & Correlation coefficient & $\rho_{s v}=0.5$ \\
Correlation coefficient & $\rho_{s v}=0.5$ & Correlation coefficient & $\rho_{1 v}=-0.5$ \\
Interest rate & $\mathrm{r}=0.03$ & Correlation coefficient & $\rho_{3 v}=-0.5$ \\
\hline
\end{tabular}

TABLE 2: parameter values.

\begin{tabular}{cccccc}
\hline$\lambda_{1}$ & $\mu_{1}$ & $\delta_{1}$ & $\lambda_{2}$ & $\mu_{2}$ & \\
\hline 1 & 0 & 0.1 & 1 & 0 & 0.1 \\
\hline
\end{tabular}

TABLE 3: parameter values.

\begin{tabular}{llcccccc}
\hline$\lambda_{1}$ & $p_{1}$ & $\beta_{11}$ & $\beta_{12}$ & $\lambda_{2}$ & $p_{2}$ & $\beta_{21}$ & $\beta_{22}$ \\
\hline 1 & 0.5 & 5 & 5 & 1 & 0.4 & 10 & 10 \\
\hline
\end{tabular}

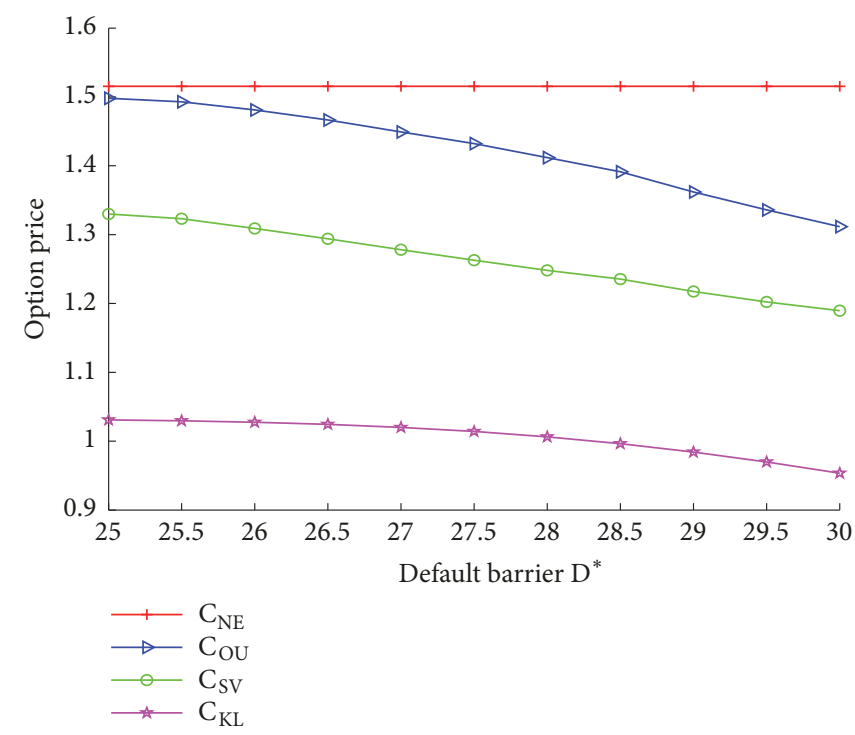

Figure 1: The effect of $D^{*}$ on the vulnerable option prices.

events happen, the option holder will only get a fraction of promised payoff; thus, the vulnerable option is less valuable.

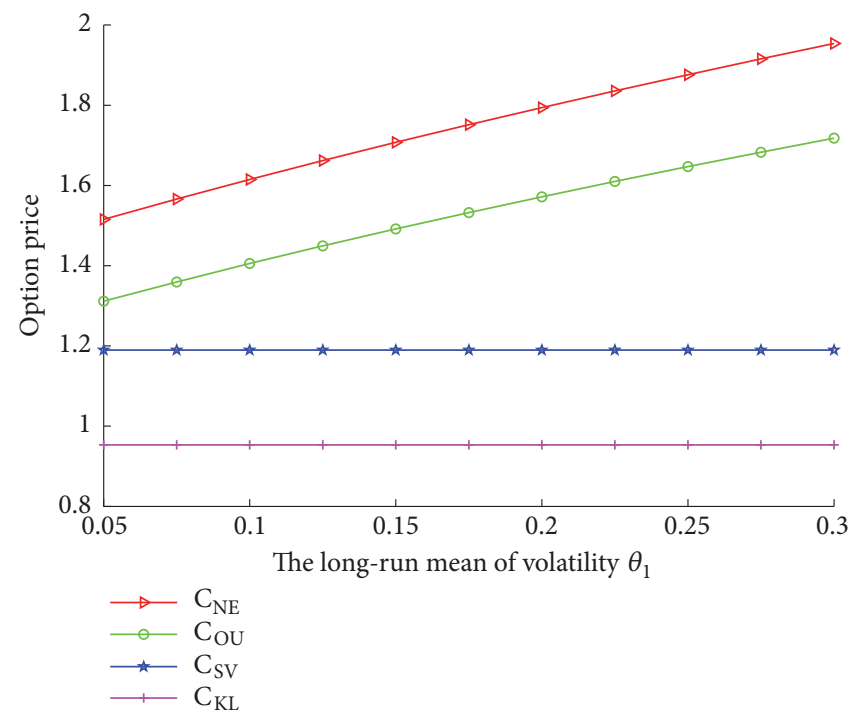

FIgUre 2: The effect of long-run mean of stochastic volatility $\theta_{1}$ on the option prices.

Figure 2 displays that the value of option price with stochastic volatility increases with the long-run mean of the process $Z_{1}(t)$. The reason for this is that a higher value $\theta_{1}$ 


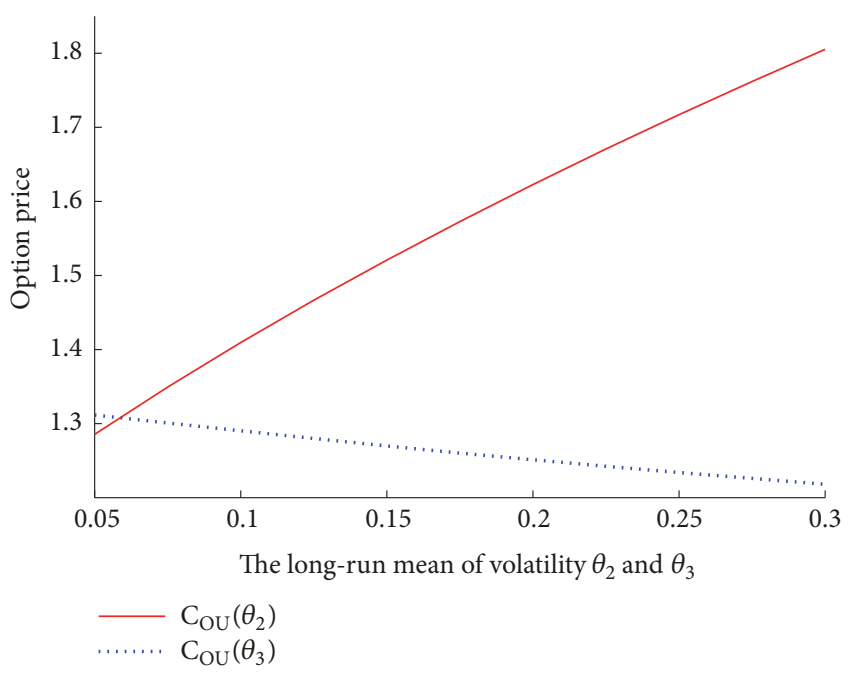

FIGURE 3: The effects of long-run means of stochastic volatility $\theta_{2}$ and $\theta_{3}$ on the vulnerable option prices.

yields a higher value of $Z_{1}(t)$. Large $Z_{1}(t)$ will increase probability that the underlying asset price and counterparty's asset value will become very low or very high at maturity; when the underlying asset price and counterparty's asset value increase, the option holder will gain benefit; when underlying asset price and counterparty's asset value decrease, the option holder faces a limited loss. Therefore the option becomes valuable if $\theta_{1}$ increases.

Figure 3 plots the effect of the long-term mean of stochastic volatility on vulnerable option price. The solid and dotted lines correspond to the vulnerable option prices obtained by the proposed model against long-run means of shortterm variances $\theta_{2}$ and $\theta_{3}$, respectively. For parameter $\theta_{2}$, we can observe that $\theta_{2}$ exerts positive effect on the vulnerable option price. The reason is the same as parameter $\theta_{1}$. Large long-run mean of variance $\theta_{2}$ will increase the probability that underlying asset price turns into very high or very low at maturity. Consequently, the vulnerable option price is valuable under large $\theta_{2}$. For parameter $\theta_{3}$, the large long-run mean of variance $\theta_{3}$ will lead to counterparty's asset value being very high or very low at maturity. Consequently, the default probability will increase. Thus, the vulnerable option price decreases with $\theta_{3}$.

As shown in Figure 4, the value of option with jump risk increases with the jump intensity $\lambda_{1}$. The reason is the same as that of $\theta_{2}$. The jump intensity will result in underlying asset price doing very poorly and very well at maturity, but the option holder has a limited loss. Thus, the option with jump risk is valuable under large jump intensity $\lambda_{1}$. Figure 5 shows that the jump intensity $\lambda_{2}$ has a negative effect on the value of vulnerable option with jump risk. Counterparty credit risk is related to the jump intensity of the counterparty's asset. A stronger jump intensity $\lambda_{2}$ of counterparty' asset value corresponds to a larger default probability. Accordingly, the vulnerable option price decreases with jump intensity $\lambda_{2}$. In addition, the vulnerable option price is a decreasing function of $\gamma$, the deadweight cost coefficient $\gamma$ affects the recovery rate when default event happens, and a higher value of $\gamma$ gives rise to a smaller recovery rate. Thus, the option holder suffer a greater loss under large $\gamma$.

4.2. The Double Exponential Jump Model. When jump components of underlying asset and counterparty's asset follow compound Poisson processes, we assume that the jump magnitude of asset price has asymmetric double exponential distribution. The Lévy kernels are given by

$$
\begin{array}{r}
v_{y}(d y) \\
=\lambda_{1}\left(p_{1} \beta_{11} e^{-\beta_{11} y} \mathbf{1}_{\{y \geq 0\}}+q_{1} \beta_{12} e^{\beta_{12} y} \mathbf{1}_{\{y<0\}}\right) d y, \\
\beta_{11}>1, \beta_{12}>0, p_{1}+q_{1}=1, \\
\nu_{\xi}(d \xi)=\lambda_{2}\left(p_{2} \beta_{21} e^{-\beta_{21} \xi} \mathbf{1}_{\{\xi \geq 0\}}+q_{2} \beta_{22} e^{\beta_{22} \xi} \mathbf{1}_{\{\xi<0\}}\right) d \xi, \\
\beta_{11}>1, \beta_{12}>0, p_{2}+q_{2}=1,
\end{array}
$$

where $\lambda_{j}(j=1,2)$ is the jump intensity of the asset process and $p_{j}$ and $q_{j}$ are probability of up-move jump and probability of down-move jump, respectively. $\beta_{j 1}$ and $\beta_{j 2}$ are mean of positive jumps and mean of negative jumps, respectively. Following Kou [25], the two integrals in (13) are given by

$$
\begin{aligned}
& \int_{R}\left(e^{\varphi_{1} y}-1\right) \nu_{y}(d y) \\
& =\lambda_{1}\left(\frac{p_{1} \beta_{11}}{\beta_{11}-\varphi_{1}}+\frac{q_{1} \beta_{12}}{\beta_{12}+\varphi_{1}}-1\right), \\
& \int_{R}\left(e^{\varphi_{2} \xi}-1\right) \nu_{\xi}(d \xi) \\
& =\lambda_{2}\left(\frac{p_{2} \beta_{21}}{\beta_{21}-\varphi_{2}}+\frac{q_{2} \beta_{22}}{\beta_{22}+\varphi_{2}}-1\right) .
\end{aligned}
$$

In Table 3, we will give the specimen values for the model parameters of assets' jump components. Figures 6 and 7 illustrate the effects of jump intensity $\lambda_{1}$ and jump intensity $\lambda_{2}$ on vulnerable option price.

Figures 6 and 7 illustrate the sensitivities of the vulnerable option price with respect to the jump intensity $\lambda_{1}$ and the jump intensity $\lambda_{2}$. From Figure 6, we observe that high jump intensity $\lambda_{1}$ leads to a high vulnerable option. Large jump intensity $\lambda_{1}$ will increase the chance that the underlying asset price will become very low or very high; the option holder will benefit from the price increase. Figure 7 shows that the vulnerable option price is a decreasing function of $\lambda_{2}$. Large jump intensity $\lambda_{2}$ will increase default probability. Therefore the vulnerable option price becomes less valuable if $\lambda_{2}$ increases. 


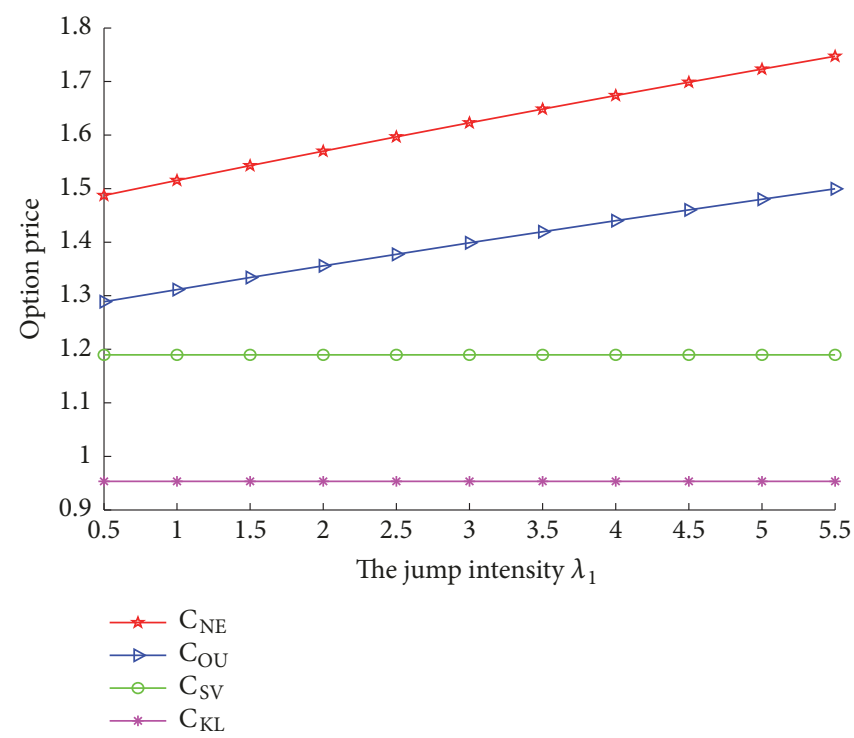

Figure 4: The effect of the jump intensity $\lambda_{1}$ on the option prices.

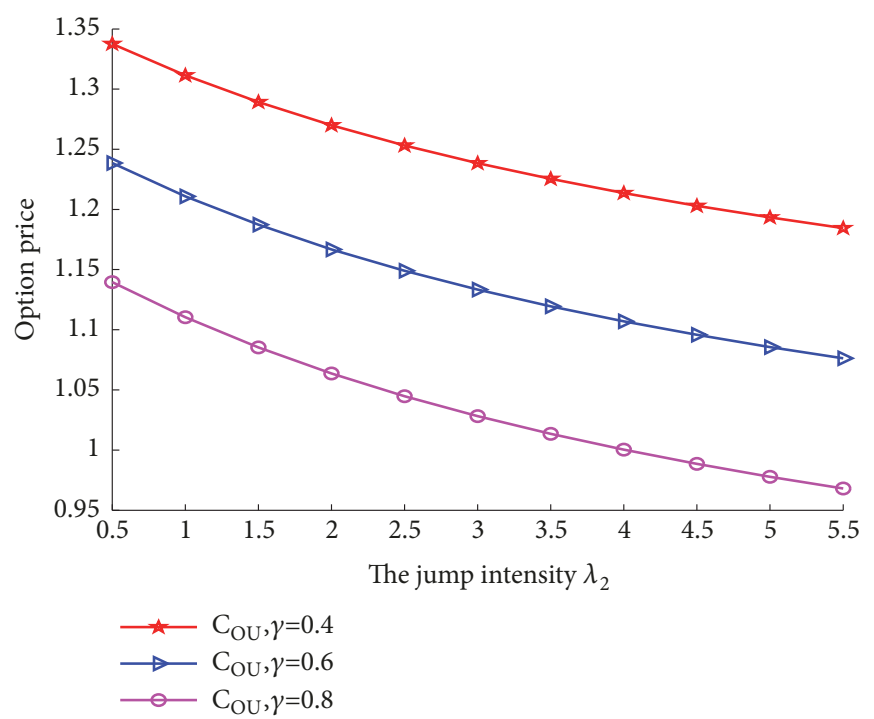

FIGURE 5: The effect of the jump intensity $\lambda_{2}$ on the vulnerable option prices.

4.3. The CGMY Model. A more general case can be considered if jump components of underlying asset and counterparty's asset follow CGMY model developed by Carr et al. [26], and the Lévy kernels are given by

$$
\begin{gathered}
\nu_{y}(y)= \begin{cases}C_{1} \frac{e^{-G_{1}|y|}}{|y|^{1+Y_{1}}}, & y<0, \\
C_{1} \frac{e^{-M_{1}|y|}}{|y|^{1+Y_{1}}}, & y>0,\end{cases} \\
\nu_{\xi}(\xi)= \begin{cases}C_{2} \frac{e^{-G_{2}|\xi|}}{|\xi|^{1+Y_{2}}}, & \xi<0, \\
C_{2} \frac{e^{-M_{2}|\xi|}}{|\xi|^{1+Y_{2}}}, & \xi>0,\end{cases}
\end{gathered}
$$

where $C_{j}>0, G_{j}>0, M_{j}>0$, and $Y_{j}<2(j=1,2) . C_{j}$ determines the aggregate activity rate of the CGMY model while the parameters $G_{j}$ and $M_{j}$ determine the height of the upward and downward jumps, respectively. $Y_{j}$ controls both tails of the spot return distribution. Based on this, following Carr et al. [26], the two integrals in (13) are given by

$$
\begin{aligned}
& \int_{R}\left(e^{\varphi_{1} y}-1\right) v_{y}(d y)=C_{1} \Gamma\left(-Y_{1}\right) \\
& \cdot\left[\left(M_{1}-\varphi_{1}\right)^{Y_{1}}-M_{1}^{Y_{1}}+\left(G_{1}+\varphi_{1}\right)^{Y_{1}}-G_{1}^{Y_{1}}\right], \\
& \int_{R}\left(e^{\varphi_{2} \xi}-1\right) \nu_{\xi}(d \xi)=C_{2} \Gamma\left(-Y_{2}\right) \\
& \cdot\left[\left(M_{2}-\varphi_{2}\right)^{Y_{2}}-M_{2}^{Y_{2}}+\left(G_{2}+\varphi_{2}\right)^{Y_{2}}-G_{2}^{Y_{2}}\right] .
\end{aligned}
$$




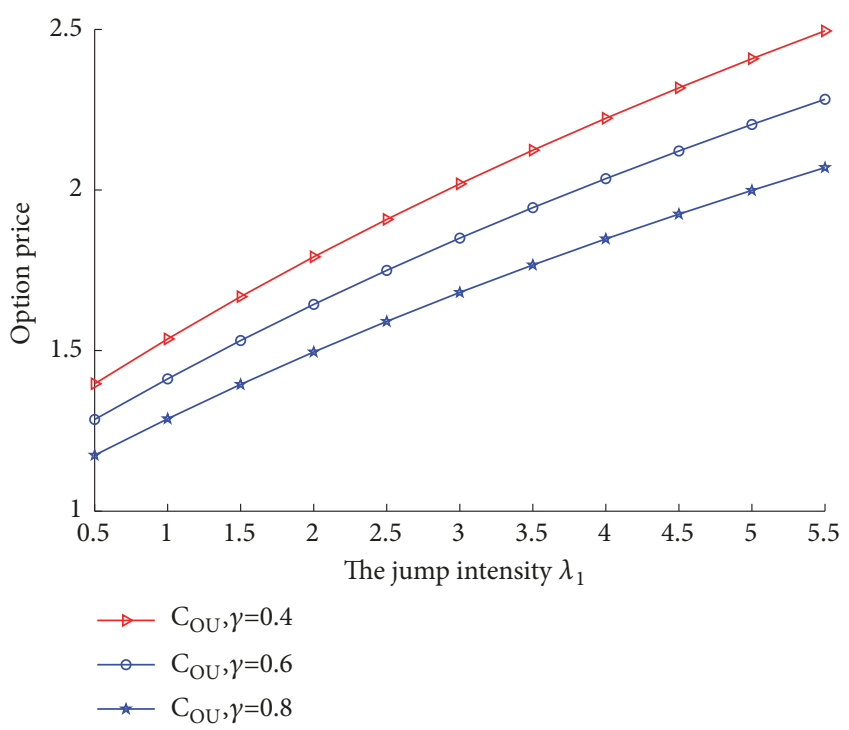

Figure 6: The effect of the jump intensity $\lambda_{1}$ on the vulnerable option prices.

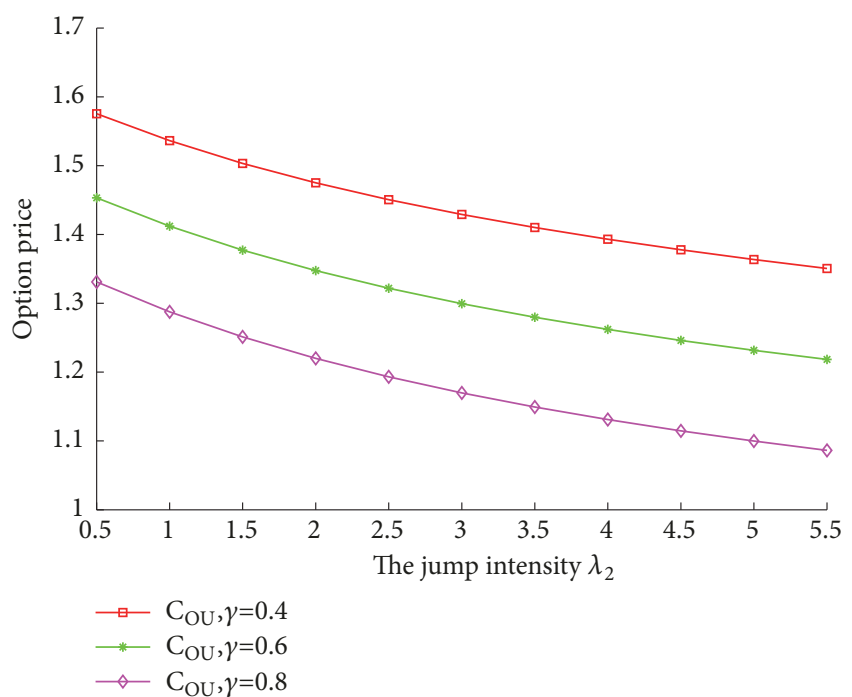

Figure 7: The effect of the jump intensity $\lambda_{2}$ on the vulnerable option prices.

In Table 4, we will give the specimen values for the model parameters of jump parts of underlying asset price and counterparty's asset value. Figures 8-11 illustrate the effects of basic parameters on vulnerable option prices. We denote prices of nonvulnerable European option in Li et al. [20] by $C_{N V}$.

From Figure 8, we can see that the value of option with jumps increases with parameter $C_{1}$. The parameter $C_{1}$ determines the aggregate activity rate of jumps of the underlying asset price; the large jump frequency will lead to the underlying asset price doing either very well or very poorly at maturity. While the option holder has limited downside risk, the vulnerable option becomes valuable if $C_{1}$ increases. As shown in Figure 9, the price of option with jumps is

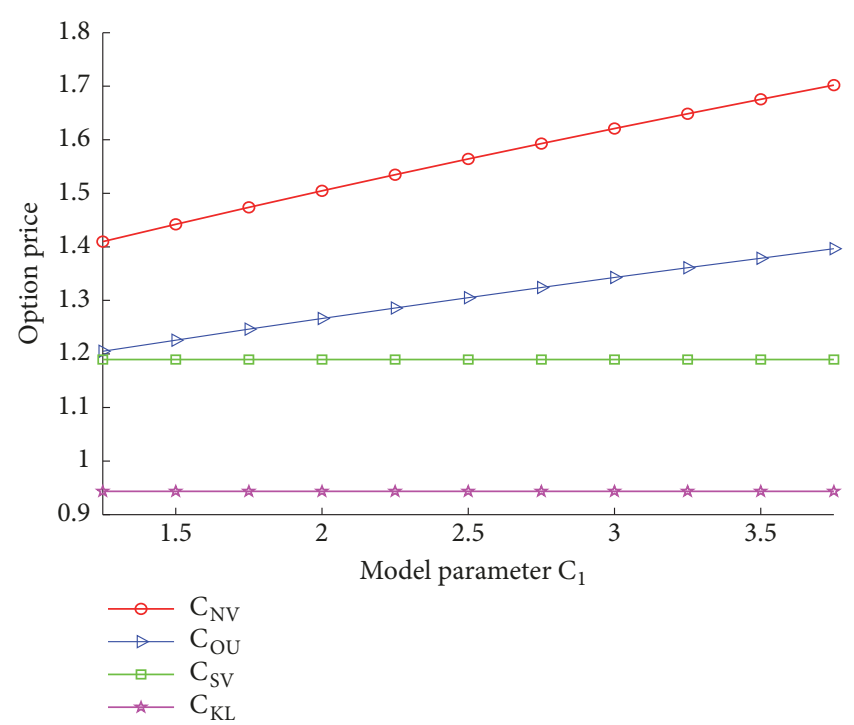

Figure 8: The effect of $C_{1}$ on the option prices.

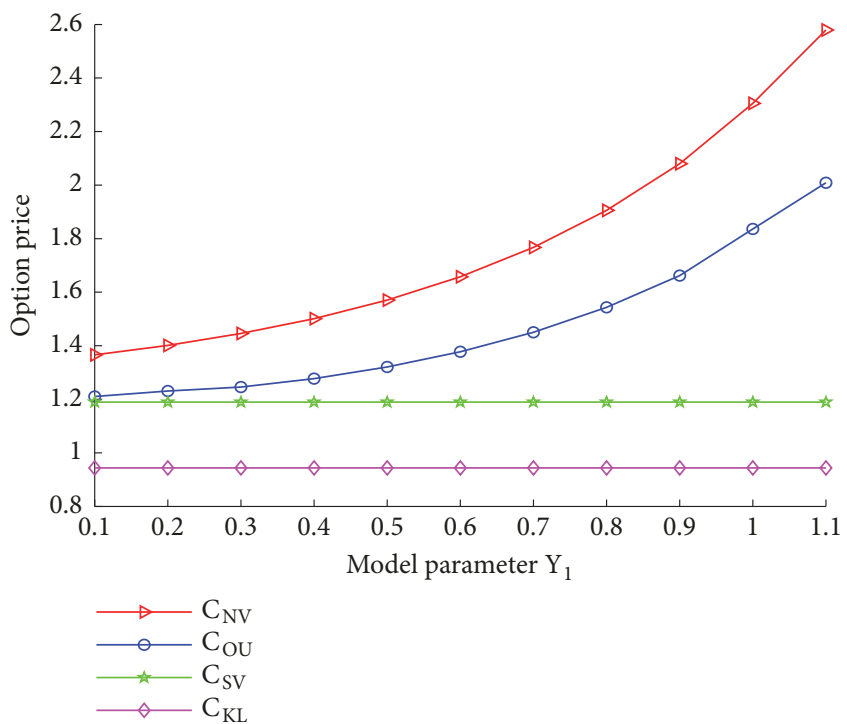

FIgURE 9: The effect of $Y_{1}$ on the option prices.

a monotonic increasing function of $Y_{1}$. The parameter $Y_{1}$ controls both tails of the underlying return distribution. As $Y_{1}$ increases, both tails of underlying return distribution will become fatter, and the underlying asset price becomes very high at maturity; it is not surprising that the option with jumps would become more valuable if $Y_{1}$ increases.

Figure 10 denotes vulnerable option price is a decreasing function of parameter $C_{2}$. The parameter $C_{2}$ controls the aggregate activity rate of jumps of the counterparty's asset value. Large $C_{2}$ will increase the probability that counterparty's asset value becomes very low at maturity, which implies that the default probability will increase. Thus, the vulnerable option price will decrease with $C_{2}$. Figure 11 shows that effect of parameter $Y_{2}$ on the vulnerable option price; the 
TABLE 4: parameter values of CGMY model.

\begin{tabular}{cccccccc}
\hline$C_{1}$ & $G_{1}$ & $M_{1}$ & $Y_{1}$ & $C_{2}$ & $G_{2}$ & $M_{2}$ & \\
\hline 1.5 & 12 & 25 & 0.25 & 1 & 13 & 22 & 0.2 \\
\hline
\end{tabular}

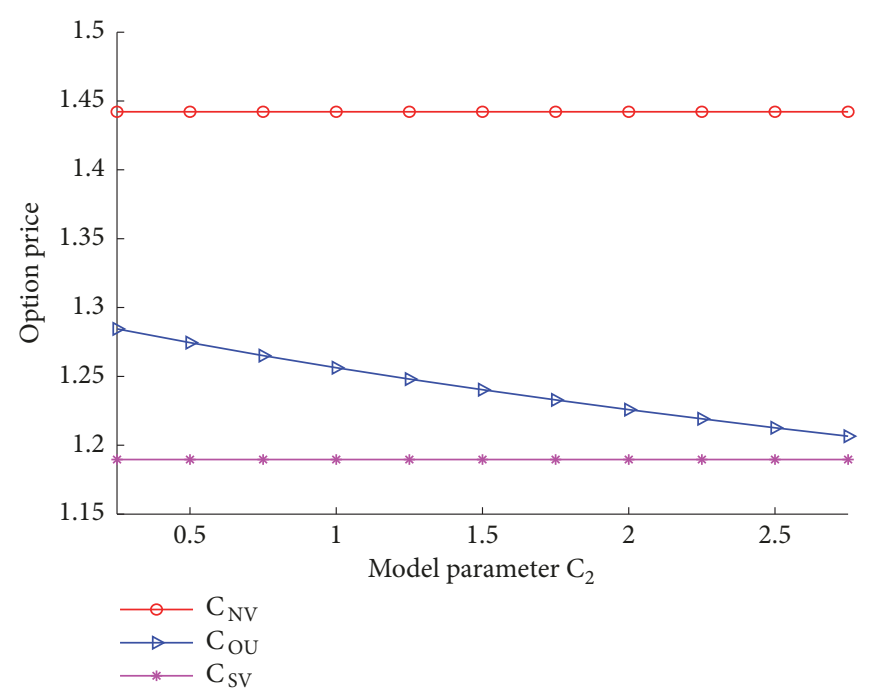

FIGURE 10: The effect of $C_{2}$ on the vulnerable option prices.

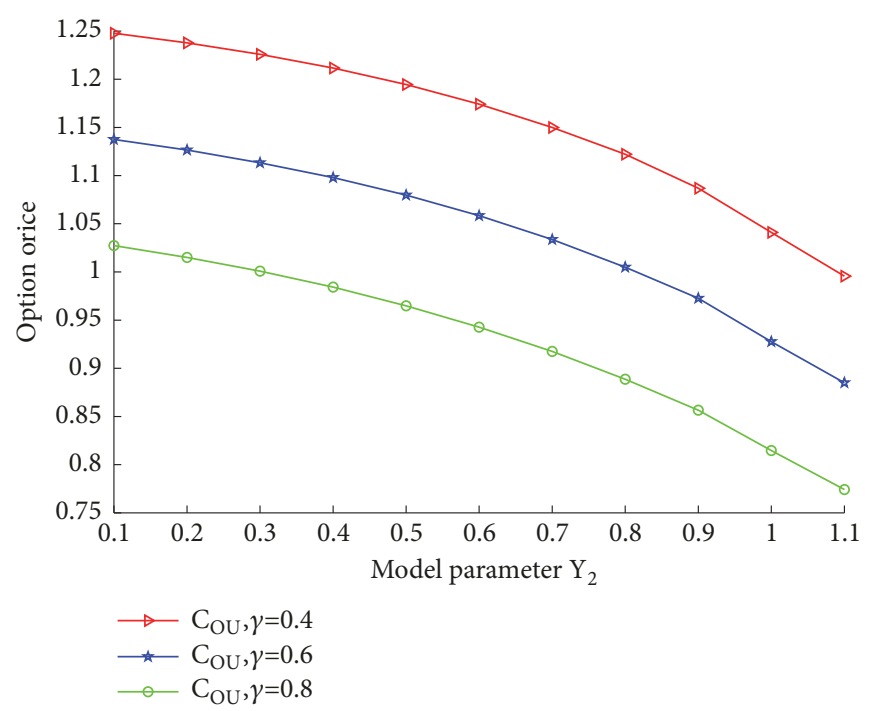

FIgURE 11: The effect of $Y_{2}$ on the vulnerable option prices.

parameter $Y_{2}$ controls both tails of the spot return distribution of counterparty's asset. As $Y_{2}$ increases, default probability will increase. Accordingly, the vulnerable option price is likely to decrease with $Y_{2}$.

\section{Conclusion}

This paper investigates the pricing problem of vulnerable European call options where the price processes of underlying asset and counterparty's asset are governed by two correlated exponential Lévy processes with stochastic volatility. We divide volatility risk of underlying asset and counterparty's asset into long-term and short-term risk. A mean-reverting process is introduced to allow for the common long-term volatility risk in the underlying asset price and counterparty's asset value. The short-term fluctuation of stochastic volatility also follows a mean-reverting process. Based on the proposed model, we explicitly obtain the joint moment generating function of underlying log-asset price and counter's log-asset value. By using the Fourier inversion transform, an analytic solution of vulnerable European call option is derived. Finally, sensitivity analysis and numerical simulation are provided to illustrate the effects of model parameters on the option price. The main findings are as follows: (i) the long-run 
means of common long-term variance of underlying asset and counterparty's asset have positive effect on the vulnerable option price. A large long-run mean of common long-term variance will increase the possibility that underlying asset price and counterparty's asset value will become very well or very poor at maturity. The option holder will benefit form increase of underlying asset price and faces a limited loss. Therefore the vulnerable option becomes valuable. (ii) The value of vulnerable option price increases with long-run mean of short-term variance of underlying asset price and decreases with long-run mean of short-term variance of counterparty' asset value; (iii) the value in proposed model is higher than those of Wang et al. [13] and Klein [5]. The jump components of underlying asset price will increase the probability that the underlying asset price will turn to very low or very high at maturity, and the vulnerable option with jump risk becomes valuable; (iv) when the jump components of underlying asset price and counterparty' asset value follow the CGMY model, the value of option price increases with the aggregate activity rate of jumps of underlying asset price and decreases with the aggregate activity rate of jumps of counterparty's asset value.

However, two further extensions of vulnerable European option pricing would be interesting. One possible extension is to incorporate stochastic interest into pricing problem of vulnerable option. Another extension would be to extend the study on vulnerable option pricing under Markov regimeswitching setting.

\section{Appendix}

\section{A. Proof of Lemma 1}

To obtain the joint moment generating function for the logasset price $\ln S(T)$ and the log-assets value $\ln V(T)$, we first take the following transformation:

$$
\begin{aligned}
& d \ln S(t)=\left(r-\int_{R}\left(e^{y}-1\right) v_{y}(d y)\right. \\
& \left.-\frac{1}{2}\left(\eta_{1}^{2} Z_{1}(t)+Z_{2}(t)\right)\right) d t \\
& +\eta_{1} \sqrt{Z_{1}(t)} d W_{1}^{S}(t)+\sqrt{Z_{2}(t)} d W_{2}^{S}(t) \\
& +\int_{R} y d N_{y}(d t, d y), \\
& d \ln V(t)=\left(r-\int_{R}\left(e^{\xi}-1\right) v_{\xi}(d \xi)\right. \\
& \left.-\frac{1}{2}\left(\eta_{2}^{2} Z_{1}(t)+Z_{3}(t)\right)\right) d t \\
& +\eta_{2} \sqrt{Z_{1}(t)} d W_{1}^{V}(t)+\sqrt{Z_{3}(t)} d W_{3}^{V}(t) \\
& +\int_{R} \xi N_{\xi}(d t, d \xi) .
\end{aligned}
$$

Let $\ln S(t)=x_{1}, \ln V(t)=x_{2}, Z_{1}(t)=z_{1}, Z_{2}(t)=z_{2}$, and $Z_{3}(t)=z_{3}$, and the generalized Feynman-Kac theorem implies that $f\left(x_{1}, x_{2}, z_{1}, z_{2}, z_{3}, \tau ; \varphi_{1}, \varphi_{2}\right)$ solves the following partial integrodifferential equation (PIDE):

$$
\begin{aligned}
& -\frac{\partial f}{\partial \tau}+\left(r-\int_{R}\left(e^{y}-1\right) v_{y}(d y)\right. \\
& \left.-\frac{1}{2}\left(\eta_{1}^{2} z_{1}+z_{2}\right)\right) \frac{\partial f}{\partial x_{1}}+\frac{1}{2}\left(\eta_{1}^{2} z_{1}+z_{2}\right) \frac{\partial^{2} f}{\partial x_{1}^{2}} \\
& +\sum_{j=1}^{3}\left[\kappa_{j}\left(\theta_{j}-z_{j}\right) \frac{\partial f}{\partial z_{j}}+\frac{1}{2} \sigma_{j}^{2} z_{j} \frac{\partial^{2} f}{\partial z_{j}^{2}}\right]+(r \\
& \left.-\int_{R}\left(e^{\xi}-1\right) \nu_{\xi}(d \xi)-\frac{1}{2}\left(\eta_{2}^{2} z_{1}+z_{3}\right)\right) \frac{\partial f}{\partial x_{2}} \\
& \left.+\frac{1}{2}\left(\eta_{2}^{2} z_{1}+z_{3}\right)\right) \frac{\partial^{2} f}{\partial x_{2}^{2}}+\eta_{1} \sigma_{1} \rho_{1 s} z_{1} \frac{\partial^{2} f}{\partial x_{1} \partial z_{1}} \\
& +\sigma_{2} \rho_{2 s} z_{2} \frac{\partial^{2} f}{\partial x_{1} \partial z_{2}}+\eta_{2} \sigma_{1} \rho_{1 v} z_{1} \frac{\partial^{2} f}{\partial x_{2} \partial z_{1}} \\
& +\sigma_{3} \rho_{3 v} z_{3} \frac{\partial^{2} f}{\partial x_{2} \partial z_{3}}+\eta_{1} \eta_{2} \rho_{s v} z_{1} \frac{\partial^{2} f}{\partial x_{1} \partial x_{2}} \\
& +\int_{R}\left[f\left(x_{1}+y, x_{2}, z_{1}, z_{2}, z_{3}, \tau ; \varphi_{1}, \varphi_{2}\right)-f\right] \\
& \cdot v_{y} d(y) \\
& +\int_{R}\left[f\left(x_{1}, x_{2}+\xi, z_{1}, z_{2}, z_{3}, \tau ; \varphi_{1}, \varphi_{2}\right)-f\right] \\
& \cdot \nu_{\xi} d(\xi)=0 \text {. }
\end{aligned}
$$

The boundary condition for (A.3) is given by

$$
f\left(x_{1}, x_{2}, 0 ; \varphi_{1}, \varphi_{2}\right)=e^{\varphi_{1} \ln S(T)+\varphi_{2} \ln V(T)} .
$$

This PDE has an exponential-affine solution of the form

$$
\begin{aligned}
f & \left(x_{1}, x_{2}, \tau ; \varphi_{1}, \varphi_{2}\right) \\
& =e^{\varphi_{1} x_{1}+\varphi_{2} x_{2}+A(\tau) z_{1}+B(\tau) z_{2}+D(\tau) z_{3}+E(\tau)},
\end{aligned}
$$

with the boundary conditions

$$
A(0)=B(0)=D(0)=E(0)=0 .
$$

Substituting (A.5) into PDE in (A.3) yields

$$
\begin{aligned}
-\left(A_{\tau}(\tau) z_{1}+B_{\tau}(\tau) z_{2}+D_{\tau}(\tau) z_{3}+E_{\tau}(\tau)\right) \\
+\left(r-\int_{R}\left(e^{y}-1\right) v_{y}(d y)-\frac{1}{2}\left(\eta_{1}^{2} z_{1}+z_{2}\right)\right) \varphi_{1} \\
\quad+\frac{1}{2}\left(\eta_{1}^{2} z_{1}+z_{2}\right) \varphi_{1}^{2} \\
+\left(r-\int_{R}\left(e^{\xi}-1\right) \nu_{\xi}(d \xi)-\frac{1}{2}\left(\eta_{2}^{2} z_{1}+z_{3}\right)\right) \varphi_{2} \\
+\frac{1}{2}\left(\eta_{2}^{2} z_{1}+z_{3}\right) \varphi_{2}^{2}+\kappa_{1}\left(\theta_{1}-z_{1}\right) A(\tau) \\
+\frac{1}{2} \sigma_{1}^{2} z_{1} A^{2}(\tau)+\kappa_{2}\left(\theta_{2}-z_{2}\right) B(\tau)
\end{aligned}
$$




$$
\begin{aligned}
& +\frac{1}{2} \sigma_{2}^{2} z_{2} B^{2}(\tau)+\kappa_{3}\left(\theta_{3}-z_{3}\right) D(\tau) \\
& +\frac{1}{2} \sigma_{3}^{2} z_{3} D^{2}(\tau)+\eta_{1} \rho_{1 s} \sigma_{1} \varphi_{1} z_{1} A(\tau) \\
& +\sigma_{2} \rho_{2 s} \varphi_{1} z_{2} B(\tau)+\eta_{2} \rho_{1 v} \sigma_{1} \varphi_{2} z_{1} A(\tau) \\
& +\rho_{3 v} \sigma_{3} \varphi_{2} z_{3} D(\tau)+\eta_{1} \eta_{2} \rho_{s v} \varphi_{1} \varphi_{2} z_{1} \\
& +\int_{R}\left(e^{\varphi_{1} y}-1\right) \nu_{y} d(y)+\int_{R}\left(e^{\varphi_{2} \xi}-1\right) \nu_{\xi} d(\xi) \\
& =0 .
\end{aligned}
$$

By matching coefficients, we can derive the following three ordinary differential equations (ODEs):

$$
\begin{aligned}
& A_{\tau}(\tau)=\frac{1}{2} \sigma_{1}^{2} A(\tau)^{2}+\left(\eta_{1} \rho_{1 s} \sigma_{1} \varphi_{1}+\eta_{2} \rho_{1 v} \sigma_{1} \varphi_{2}\right. \\
& \left.\quad-\kappa_{1}\right) A(\tau)-\frac{1}{2}\left(\eta_{1}^{2} \varphi_{1}+\eta_{2}^{2} \varphi_{2}-\eta_{1}^{2} \varphi_{1}^{2}-\eta_{2}^{2} \varphi_{2}^{2}\right. \\
& \left.\quad-2 \eta_{1} \eta_{2} \rho_{s v} \varphi_{1} \varphi_{2}\right) \\
& B_{\tau}(\tau)=\frac{1}{2} \sigma_{2}^{2} B(\tau)^{2}+\left(\sigma_{2} \rho_{2 s} \varphi_{1}-\kappa_{2}\right) B(\tau)-\frac{1}{2}\left(\varphi_{1}\right. \\
& \left.\quad-\varphi_{1}^{2}\right) \\
& D_{\tau}(\tau)=\frac{1}{2} \sigma_{3}^{2} D(\tau)^{2}+\left(\sigma_{3} \rho_{3 v} \varphi_{2}-\kappa_{3}\right) D(\tau)-\frac{1}{2}\left(\varphi_{2}\right. \\
& \left.\quad-\varphi_{2}^{2}\right) \\
& E_{\tau}(\tau)=r\left(\varphi_{1}+\varphi_{2}\right) \\
& \quad+\int_{R}\left[e^{\varphi_{1} y}-1-\varphi_{1}\left(e^{y}-1\right)\right] \nu_{y} d(y) \\
& \quad+\int_{R}\left[e^{\varphi_{2} \xi}-1-\varphi_{2}\left(e^{\xi}-1\right)\right] \nu_{\xi} d(\xi)+\kappa_{1} \theta_{1} A(\tau) \\
& \quad+\kappa_{2} \theta_{2} B(\tau)+\kappa_{3} \theta_{3} D(\tau) .
\end{aligned}
$$

According to the boundary condition (A.6), the above ODEs can be easily solved as below:

$$
\begin{aligned}
& A(\tau)=-\frac{a_{1}\left(1-e^{-\Delta_{1} \tau}\right)}{2 \Delta_{1}-\left(\Delta_{1}+b_{1}\right)\left(1-e^{-\Delta_{1} \tau}\right)}, \\
& B(\tau)=-\frac{a_{2}\left(1-e^{-\Delta_{2} \tau}\right)}{2 \Delta_{2}-\left(\Delta_{2}+b_{2}\right)\left(1-e^{-\Delta_{2} \tau}\right)}, \\
& D(\tau)=-\frac{a_{3}\left(1-e^{-\Delta_{3} \tau}\right)}{2 \Delta_{3}-\left(\Delta_{3}+b_{3}\right)\left(1-e^{-\Delta_{3} \tau}\right)}, \\
& E(\tau)=\left(r\left(\varphi_{1}+\varphi_{2}\right)\right. \\
& +\int_{R}\left[e^{\varphi_{1} y}-1-\varphi_{1}\left(e^{y}-1\right)\right] \nu_{y} d(y)
\end{aligned}
$$

$$
\begin{aligned}
& \left.+\int_{R}\left[e^{\varphi_{2} \xi}-1-\varphi_{2}\left(e^{\xi}-1\right)\right] \nu_{\xi} d(\xi)\right) \tau \\
& -\frac{\kappa_{1} \theta_{1}}{\sigma_{1}^{2}}\left(\left(\Delta_{1}+b_{1}\right) \tau\right. \\
& \left.+2 \ln \left[1-\frac{\Delta_{1}+b_{1}}{2 \Delta_{1}}\left(1-e^{-\Delta_{1} \tau}\right)\right]\right) \\
& -\frac{\kappa_{2} \theta_{2}}{\sigma_{2}^{2}}\left(\left(\Delta_{2}+b_{2}\right) \tau\right. \\
& \left.+2 \ln \left[1-\frac{\Delta_{2}+b_{2}}{2 \Delta_{1}}\left(1-e^{-\Delta_{2} \tau}\right)\right]\right) \\
& -\frac{\kappa_{3} \theta_{3}}{\sigma_{3}^{2}}\left(\left(\Delta_{3}+b_{3}\right) \tau\right. \\
& \left.+2 \ln \left[1-\frac{\Delta_{3}+b_{3}}{2 \Delta_{3}}\left(1-e^{-\Delta_{3} \tau}\right)\right]\right)
\end{aligned}
$$

where

$$
\begin{aligned}
& \Delta_{1}=\sqrt{b_{1}^{2}+\sigma_{1}^{2} a_{1}} \\
& a_{1}=\left(\eta_{1}^{2} \varphi_{1}+\eta_{2}^{2} \varphi_{2}-\eta_{1}^{2} \varphi_{1}^{2}-\eta_{2}^{2} \varphi_{2}^{2}-2 \eta_{1} \eta_{2} \rho_{s v} \varphi_{1} \varphi_{2}\right) \\
& b_{1}=\left(\eta_{1} \rho_{1 s} \sigma_{1} \varphi_{1}+\eta_{2} \rho_{1 v} \sigma_{1} \varphi_{2}-\kappa_{1}\right) \\
& \Delta_{2}=\sqrt{b_{2}^{2}+\sigma_{2}^{2} a_{2}} . \\
& a_{2}=\left(\varphi_{1}-\varphi_{1}^{2}\right) \\
& b_{2}=\left(\varphi_{1} \sigma_{2} \rho_{2 s}-\kappa_{2}\right) \\
& \Delta_{3}=\sqrt{b_{3}^{2}+\sigma_{3}^{2} a_{3}} \\
& a_{3}=\left(\varphi_{2}-\varphi_{2}^{2}\right) \\
& b_{3}=\left(\varphi_{2} \sigma_{3} \rho_{3 v}-\kappa_{3}\right)
\end{aligned}
$$

\section{B. Proof of Theorem 2}

Proof. Recall the joint moment generating function of $\ln S_{1}(T)$ and $\ln V(T)$ in Lemma 1 ,

$$
f\left(x_{1}, x_{2}, \tau ; \varphi_{1}, \varphi_{2}\right)=E^{Q}\left[e^{\varphi_{1} \ln S(T)+\varphi_{2} \ln V(T)} \mid \mathscr{F}_{t}\right] .
$$

To deal with the term $C_{1}(t, T)$ in (16), we define a new probability measure $Q_{1}$ on $\mathscr{F}_{T}$ by the following RandonNikodym derivative:

$$
\left.\frac{d Q_{1}}{d Q}\right|_{\mathscr{F}_{T}}=\frac{S(T)}{E^{Q}\left[S(T) \mid \mathscr{F}_{t}\right]} .
$$

Based on the definition of $Q_{1}$, we have the characteristic function of $\ln S(T)$ and $\ln V(T)$ under $Q_{1}$, 


$$
\begin{aligned}
f_{1}\left(\tau, i \varphi_{1}, i \varphi_{2}\right) & =E^{Q_{1}}\left[e^{i \varphi_{1} \ln S(T)+i \varphi_{2} \ln V(T)} \mid \mathscr{F}_{t}\right] \\
& =\frac{E^{\mathrm{Q}}\left[e^{i \varphi_{1} \ln S(T)+i \varphi_{2} \ln V(T)} S(T) \mid \mathscr{F}_{t}\right]}{E^{\mathrm{Q}}\left[S(T) \mid \mathscr{F}_{t}\right]} \\
& =\frac{E^{\mathrm{Q}}\left[e^{\left(i \varphi_{1}+1\right) \ln S(T)+i \varphi_{2} \ln V(T)} \mid \mathscr{F}_{t}\right]}{E^{\mathrm{Q}}\left[e^{\ln S(T)} \mid \mathscr{F}_{t}\right]} \\
& =\frac{f\left(x_{1}, x_{2}, \tau ; i \varphi_{1}+1, i \varphi_{2}\right)}{f\left(x_{1}, x_{2}, \tau ; 1,0\right)},
\end{aligned}
$$

where we have used the definition of $f\left(x_{1}, x_{2}, \tau ; \varphi_{1}, \varphi_{2}\right)$ in Appendix A.

From standard probability theory (see, e.g., Kendall and Stuart [27] and Shephard [28]), one obtains that the distribution function $F_{1}\left(\ln S(T), \ln V(T) ; k_{1}, k_{2}\right)$ corresponding to the joint characteristic function $f_{1}\left(\tau, i \varphi_{1}, i \varphi_{2}\right)$ is given by

$$
\begin{gathered}
F_{1}\left(\ln S(T), \ln V(T) ; k_{1}, k_{2}\right)=-\frac{1}{4}+\frac{1}{2} F_{1}\left(\ln S(T) ; k_{1}\right) \\
+\frac{1}{2} F_{1}\left(\ln V(T) ; k_{2}\right)-\frac{1}{2 \pi^{2}} \\
\cdot \int_{0}^{\infty} \int_{0}^{\infty}\left(\operatorname{Re}\left[\frac{e^{-i \varphi_{1} k_{1}-i \varphi_{2} k_{2}} f_{1}\left(\tau, i \varphi_{1}, i \varphi_{2}\right)}{\varphi_{1} \varphi_{2}}\right]\right. \\
\left.-\operatorname{Re}\left[\frac{e^{-i \varphi_{1} k_{1}+i \varphi_{2} k_{2}} f_{1}\left(\tau, i \varphi_{1},-i \varphi_{2}\right)}{\varphi_{1} \varphi_{2}}\right]\right) d \varphi_{1} d \varphi_{2}
\end{gathered}
$$

where $\mathrm{Re}[\cdot]$ represents real part of a complex number, and $F_{1}\left(\ln S(T) ; k_{1}\right)$ and $F_{1}\left(\ln V(T) ; k_{2}\right)$ are the marginal distributions for $\ln S(T)$ and $\ln V(T)$,

$$
\begin{aligned}
& F_{1}\left(\ln S(T) ; k_{1}\right) \\
& =\frac{1}{2}-\frac{1}{\pi} \int_{0}^{\infty} \operatorname{Re}\left[\frac{e^{-i \varphi_{1} k_{1}} f_{1}\left(\tau, i \varphi_{1}, 0\right)}{i \varphi_{1}}\right] d \varphi_{1}, \\
& F_{1}\left(\ln V(T) ; k_{2}\right) \\
& =\frac{1}{2}-\frac{1}{\pi} \int_{0}^{\infty} \operatorname{Re}\left[\frac{e^{-i \varphi_{2} k_{2}} f_{1}\left(\tau, 0, i \varphi_{2}\right)}{i \varphi_{2}}\right] d \varphi_{2} .
\end{aligned}
$$

Here, we have that

$$
\begin{aligned}
Q_{1}\left(\ln S(T) \geq \ln K, \ln V(T) \geq \ln D^{*}\right) \\
\quad=1-F_{1}(\ln S(T) ; \ln K)-F_{1}\left(\ln V(T) ; \ln D^{*}\right) \\
\quad+F_{1}\left(\ln S(T), \ln V(T) ; \ln K, \ln D^{*}\right)
\end{aligned}
$$

Recalling the definition of the probability measure $Q_{1}$, we have that

$$
\begin{aligned}
& Q_{1}\left(\ln S(T) \geq \ln K, \ln V(T) \geq \ln D^{*}\right)=\frac{1}{4}+\frac{1}{2 \pi} \\
& \cdot \int_{0}^{\infty} \operatorname{Re}\left[\frac{e^{-i \varphi_{1} \ln K} f_{1}\left(\tau, i \varphi_{1}, 0\right)}{i \varphi_{1}}\right] d \varphi_{1}+\frac{1}{2 \pi} \\
& \cdot \int_{0}^{\infty} \operatorname{Re}\left[\frac{e^{-i \varphi_{2} \ln D^{*}} f_{1}\left(\tau, 0, i \varphi_{2}\right)}{i \varphi_{2}}\right] d \varphi_{2}-\frac{1}{2 \pi^{2}} \\
& \cdot \int_{0}^{\infty} \int_{0}^{\infty}\left(\operatorname{Re}\left[\frac{e^{-i \varphi_{1} \ln K-i \varphi_{2} \ln D^{*}} f_{1}\left(\tau, i \varphi_{1}, i \varphi_{2}\right)}{\varphi_{1} \varphi_{2}}\right]\right. \\
& \left.-\operatorname{Re}\left[\frac{e^{-i \varphi_{1} \ln K+i \varphi_{2} \ln D^{*}} f_{1}\left(\tau, i \varphi_{1},-i \varphi_{2}\right)}{\varphi_{1} \varphi_{2}}\right]\right) d \varphi_{1} d \varphi_{2} .
\end{aligned}
$$

Recalling the definition of the probability measure $Q_{1}$, we have that

$$
\begin{aligned}
Q_{1} & \left(\ln S(T) \geq \ln K, \ln V(T) \geq \ln D^{*}\right) \\
= & \frac{E^{Q}\left[S(T) \mathbf{1}_{\left\{\ln S(T) \geq \ln K, \ln V(T) \geq \ln D^{*}\right\}} \mid \mathscr{F}_{t}\right]}{E^{Q}\left[S_{1}(T) \mid \mathscr{F}_{t}\right]},
\end{aligned}
$$

which in turn implies

$$
\begin{aligned}
C_{1}(t, T)= & Q_{1}\left(\ln S(T) \geq \ln K, \ln V(T) \geq \ln D^{*}\right) \\
& * E^{\mathrm{Q}}\left[S(T) \mid \mathscr{F}_{t}\right] \\
= & \Pi_{1}+\frac{1}{4} f\left(x_{1}, x_{2}, \tau ; 1,0\right),
\end{aligned}
$$

where

$$
\begin{aligned}
\Pi_{1} & =\frac{1}{2 \pi} \int_{0}^{\infty} \operatorname{Re}\left[\frac{e^{-i \varphi_{1} \ln K} f\left(x_{1}, x_{2}, \tau ; i \varphi_{1}+1,0\right)}{i \varphi_{1}}\right] d \varphi_{1}+\frac{1}{2 \pi} \int_{0}^{\infty} \operatorname{Re}\left[\frac{e^{-i \varphi_{2} \ln D^{*}} f\left(x_{1}, x_{2}, \tau ; 1, i \varphi_{2}\right)}{i \varphi_{2}}\right] d \varphi_{2}-\frac{1}{2 \pi^{2}} \\
& \cdot \int_{0}^{\infty} \int_{0}^{\infty}\left(\operatorname{Re}\left[\frac{e^{-i \varphi_{1} \ln K-i \varphi_{2} \ln D^{*}} f\left(x_{1}, x_{2}, \tau ; i \varphi_{1}+1, i \varphi_{2}\right)}{\varphi_{1} \varphi_{2}}\right]\right. \\
& \left.-\operatorname{Re}\left[\frac{e^{-i \varphi_{1} \ln K+i \varphi_{2} \ln D^{*}} f\left(x_{1}, x_{2}, \tau ; i \varphi_{1}+1,-i \varphi_{2}\right)}{\varphi_{1} \varphi_{2}}\right]\right) d \varphi_{1} d \varphi_{2} .
\end{aligned}
$$

Since we have obtained the characteristic function $f\left(x_{1}, x_{2}\right.$, $\left.\tau ; \varphi_{1}, \varphi_{2}\right)$ of $\ln S(T)$ and $\ln V(T)$ under $Q$, the corresponding distribution function $F\left(\ln S(T), \ln V(T) ; \ln K, \ln D^{*}\right)$ can be derived and hence it holds that 


$$
\begin{aligned}
& \Pi_{2}(t, T)=C_{2}(t, T)=Q\left(\ln S(T) \geq \ln K, \ln V(T) \geq \ln D^{*}\right)=1-F(\ln S(T) ; \ln K)-F\left(\ln V(T) ; \ln D^{*}\right) \\
& +F\left(\ln S(T), \ln V(T) ; \ln K, \ln D^{*}\right)=\frac{1}{4}+\frac{1}{2 \pi} \int_{0}^{\infty} \operatorname{Re}\left[\frac{e^{-i \varphi_{1} \ln K} f\left(x_{1}, x_{2}, \tau ; i \varphi_{1}, 0\right)}{i \varphi_{1}}\right] d \varphi_{1}+\frac{1}{2 \pi} \\
& \quad \int_{0}^{\infty} \operatorname{Re}\left[\frac{e^{-i \varphi_{2} \ln D^{*}} f\left(x_{1}, x_{2}, \tau ; 0, i \varphi_{2}\right)}{i \varphi_{2}}\right] d \varphi_{2}-\frac{1}{2 \pi^{2}} \\
& \quad \int_{0}^{\infty} \int_{0}^{\infty}\left(\operatorname{Re}\left[\frac{e^{-i \varphi_{1} \ln K-i \varphi_{2} \ln D^{*}} f\left(x_{1}, x_{2}, \tau ; i \varphi_{1}, i \varphi_{2}\right)}{\varphi_{1} \varphi_{2}}\right]-\operatorname{Re}\left[\frac{e^{-i \varphi_{1} \ln K+i \varphi_{2} \ln D^{*}} f\left(x_{1}, x_{2}, \tau ; i \varphi_{1},-i \varphi_{2}\right)}{\varphi_{1} \varphi_{2}}\right]\right) d \varphi_{1} d \varphi_{2} .
\end{aligned}
$$
$Q_{3}$

To derive $C_{3}(t, T)$, we define a new probability measure

$$
\left.\frac{d Q_{3}}{d Q}\right|_{\mathscr{F}_{T}}=\frac{S(T) V(T)}{E^{Q}\left[S(T) V(T) \mid \mathscr{F}_{t}\right]}
$$

and the characteristic function of $\ln S(T)$ and $\ln V(T)$ under $Q_{3}$ is given by

$$
\begin{aligned}
f_{3}\left(\tau, i \varphi_{1}, i \varphi_{2}\right)=E^{Q_{3}}\left[e^{i \varphi_{1} \ln S(T)-i \varphi_{2} \ln V(T)} \mid \mathscr{F}_{t}\right] \\
=\frac{E^{Q}\left[e^{i \varphi_{1} \ln S(T)-i \varphi_{2} \ln V(T)} S(T) V(T) \mid \mathscr{F}_{t}\right]}{E^{Q}\left[S(T) V(T) \mid \mathscr{F}_{t}\right]} \\
=\frac{E^{Q}\left[e^{\left(i \varphi_{1}+1\right) \ln S(T)+\left(1-i \varphi_{2}\right) \ln V(T)} \mid \mathscr{F}_{t}\right]}{E^{\mathrm{Q}}\left[e^{\left.\ln S(T)+\ln V(T) \mathscr{F}_{t}\right]}\right.} \\
=\frac{f\left(x_{1}, x_{2}, \tau ; i \varphi_{1}+1,1-i \varphi_{2}\right)}{f\left(x_{1}, x_{2}, \tau ; 1,1\right)} .
\end{aligned}
$$

and

$$
\begin{aligned}
C_{3}(t, T) \\
=Q_{3}\left(\ln S(T) \geq \ln K,-\ln V(T) \geq-\ln D^{*}\right) \\
\quad * E^{Q}\left[S(T) V(T) \mid \mathscr{F}_{t}\right] \\
=\Pi_{3}+\frac{1}{4} f\left(x_{1}, x_{2}, \tau ; 1,1\right),
\end{aligned}
$$

where

$Q_{3}\left(\ln S(T) \geq \ln K,-\ln V(T) \geq-\ln D^{*}\right)=\frac{1}{4}+\frac{1}{2 \pi}$

$$
\begin{aligned}
& \cdot \int_{0}^{\infty} \operatorname{Re}\left[\frac{e^{-i \varphi_{1} \ln K} f_{3}\left(\tau, i \varphi_{1}, 0\right)}{i \varphi_{1}}\right] d \varphi_{1}+\frac{1}{2 \pi} \\
& \cdot \int_{0}^{\infty} \operatorname{Re}\left[\frac{e^{i \varphi_{2} \ln D^{*}} f_{3}\left(\tau, 0, i \varphi_{2}\right)}{i \varphi_{2}}\right] d \varphi_{2}-\frac{1}{2 \pi^{2}} \\
& \cdot \int_{0}^{\infty} \int_{0}^{\infty}\left(\operatorname{Re}\left[\frac{e^{-i \varphi_{1} \ln K+i \varphi_{2} \ln D^{*}} f_{3}\left(\tau, i \varphi_{1}, i \varphi_{2}\right)}{\varphi_{1} \varphi_{2}}\right]\right. \\
& \left.-\operatorname{Re}\left[\frac{e^{-i \varphi_{1} \ln K-i \varphi_{2} \ln D^{*}} f_{3}\left(\tau, i \varphi_{1},-i \varphi_{2}\right)}{\varphi_{1} \varphi_{2}}\right]\right) d \varphi_{1} d \varphi_{2},
\end{aligned}
$$

$$
\begin{aligned}
& \Pi_{3}(t, T)=\frac{1}{2 \pi} \int_{0}^{\infty} \operatorname{Re}\left[\frac{e^{-i \varphi_{1} \ln K} f\left(x_{1}, x_{2}, \tau ; i \varphi_{1}+1,1\right)}{i \varphi_{1}}\right] d \varphi_{1}+\frac{1}{2 \pi} \int_{0}^{\infty} \operatorname{Re}\left[\frac{e^{i \varphi_{2} \ln D^{*}} f\left(x_{1}, x_{2}, \tau ; 1,1-i \varphi_{2}\right)}{i \varphi_{2}}\right] d \varphi_{2} \\
& -\frac{1}{2 \pi^{2}} \int_{0}^{\infty} \int_{0}^{\infty}\left(\operatorname{Re}\left[\frac{e^{-i \varphi_{1} \ln K+i \varphi_{2} \ln D^{*}} f\left(x_{1}, x_{2}, \tau ; i \varphi_{1}+1,1-i \varphi_{2}\right)}{\varphi_{1} \varphi_{2}}\right]\right. \\
& \left.-\operatorname{Re}\left[\frac{e^{-i \varphi_{1} \ln K-i \varphi_{2} \ln D^{*}} f\left(x_{1}, x_{2}, \tau ; i \varphi_{1}+1,1+i \varphi_{2}\right)}{\varphi_{1} \varphi_{2}}\right]\right) d \varphi_{1} d \varphi_{2} .
\end{aligned}
$$


Analogously, standard probability theory implies that

$$
\left.\frac{d Q_{4}}{d Q}\right|_{\mathscr{F}_{T}}=\frac{V(T)}{E^{Q}\left[V(T) \mid \mathscr{F}_{t}\right]} .
$$

Therefore, the characteristic function of $\ln S(T)$ and $\ln V(T)$ under $Q_{4}$ is given by

$$
\begin{aligned}
f_{4}\left(\tau, i \varphi_{1}, i \varphi_{2}\right) & =E^{\mathrm{Q}_{4}}\left[e^{i \varphi_{1} \ln S(T)-i \varphi_{2} \ln V(T)} \mid \mathscr{F}_{t}\right] \\
& =\frac{E^{\mathrm{Q}}\left[e^{i \varphi_{1} \ln S(T)-i \varphi_{2} \ln V(T)} V(T) \mid \mathscr{F}_{t}\right]}{E^{\mathrm{Q}}\left[V(T) \mid \mathscr{F}_{t}\right]} \\
& =\frac{E^{\mathrm{Q}}\left[e^{\left(i \varphi_{1}\right) \ln S(T)+\left(1-i \varphi_{2}\right) \ln V(T)} \mid \mathscr{F}_{t}\right]}{E^{\mathrm{Q}}\left[e^{\ln V(T)} \mid \mathscr{F}_{t}\right]} \\
& =\frac{f\left(x_{1}, x_{2}, \tau ; i \varphi_{1}, 1-i \varphi_{2}\right)}{f\left(x_{1}, x_{2}, \tau ; 0,1\right)} .
\end{aligned}
$$

Similarly, one gets that

$$
\begin{aligned}
& Q_{4}\left(\ln S(T) \geq \ln K,-\ln V(T) \geq-\ln D^{*}\right)=\frac{1}{4}+\frac{1}{2 \pi} \\
& \cdot \int_{0}^{\infty} \operatorname{Re}\left[\frac{e^{-i \varphi_{1} \ln K} f_{4}\left(\tau, i \varphi_{1}, 0\right)}{i \varphi_{1}}\right] d \varphi_{1}+\frac{1}{2 \pi} \\
& \cdot \int_{0}^{\infty} \operatorname{Re}\left[\frac{e^{-i \varphi_{2} \ln D^{*}} f_{4}\left(\tau, 0, i \varphi_{2}\right)}{i \varphi_{2}}\right] d \varphi_{2}-\frac{1}{2 \pi^{2}} \\
& \cdot \int_{0}^{\infty} \int_{0}^{\infty}\left(\operatorname{Re}\left[\frac{e^{-i \varphi_{1} \ln K+i \varphi_{2} \ln D^{*}} f_{4}\left(\tau, i \varphi_{1}, i \varphi_{2}\right)}{\varphi_{1} \varphi_{2}}\right]\right. \\
& \left.-\operatorname{Re}\left[\frac{e^{-i \varphi_{1} \ln K-i \varphi_{2} \ln D^{*}} f_{4}\left(\tau, i \varphi_{1},-i \varphi_{2}\right)}{\varphi_{1} \varphi_{2}}\right]\right) d \varphi_{1} d \varphi_{2},
\end{aligned}
$$

and

$$
\begin{gathered}
C_{4}(t, T)=Q_{4}\left(\ln S(T) \geq \ln K, \ln V(T) \geq \ln D^{*}\right) \\
\cdot E^{Q}\left[V(T) \mid \mathscr{F}_{t}\right]=\Pi_{4}+\frac{1}{4} f\left(x_{1}, x_{2}, \tau ; 0,1\right),
\end{gathered}
$$

where

$$
\begin{aligned}
\Pi_{4} & =\frac{1}{2 \pi} \int_{0}^{\infty} \operatorname{Re}\left[\frac{e^{-i \varphi_{1} \ln K} f\left(x_{1}, x_{2}, \tau ; i \varphi_{1}, 1\right)}{i \varphi_{1}}\right] d \varphi_{1}+\frac{1}{2 \pi} \int_{0}^{\infty} \operatorname{Re}\left[\frac{e^{i \varphi_{2} \ln D^{*}} f\left(x_{1}, x_{2}, \tau ; 0,1-i \varphi_{2}\right)}{i \varphi_{2}}\right] d \varphi_{2}-\frac{1}{2 \pi^{2}} \\
& \cdot \int_{0}^{\infty} \int_{0}^{\infty}\left(\operatorname{Re}\left[\frac{e^{-i \varphi_{1} \ln K+i \varphi_{2} \ln D^{*}} f\left(x_{1}, x_{2}, \tau ; i \varphi_{1}, 1-i \varphi_{2}\right)}{\varphi_{1} \varphi_{2}}\right]\right. \\
& \left.-\operatorname{Re}\left[\frac{e^{-i \varphi_{1} \ln K-i \varphi_{2} \ln D^{*}} f\left(x_{1}, x_{2}, \tau ; i \varphi_{1}, 1+i \varphi_{2}\right)}{\varphi_{1} \varphi_{2}}\right]\right) d \varphi_{1} d \varphi_{2} .
\end{aligned}
$$

Therefore, the price of vulnerable options is given by

$$
\begin{aligned}
& C(t, T)=e^{-r(T-t)}\left(\Pi_{1}(t, T)+\frac{1}{4} f\left(x_{1}, x_{2}, \tau, 1,0\right)\right. \\
& \left.-K \Pi_{2}(t, T)\right)+e^{-r T(-t)} \frac{(1-\gamma)}{D}\left(\Pi_{3}(t, T)\right. \\
& \quad+\frac{1}{4} f\left(x_{1}, x_{2}, \tau, 1,1\right)-K \Pi_{4}(t, T) \\
& \left.-\frac{1}{4} K f\left(x_{1}, x_{2}, \tau, 0,1\right)\right) .
\end{aligned}
$$

\section{Data Availability}

The data used to support the findings of this study are available from the corresponding author upon request.

\section{Conflicts of Interest}

The authors declare that there is no conflict of interests regarding the publication of this paper.

\section{Acknowledgments}

This research is partially supported by the National Natural Science Foundation of China under Grant Nos. 71431008, 71521061 , and 71790593.

\section{References}

[1] S. Crépey, "Bilateral counterparty risk under funding constraints-part II: CVA," Mathematical Finance, vol. 25, no. 1, pp. 23-50, 2015.

[2] M. Sayah, "Counterparty Credit Risk in OTC Derivatives under Basel III," Journal of Mathematical Finance, vol. 07, no. 01, pp. 138, 2017.

[3] M. Breton and O. Marzouk, "Evaluation of counterparty risk for derivatives with early-exercise features," Journal of Economic Dynamics \& Control, vol. 88, pp. 1-20, 2018.

[4] H. Johnson and R. Stulz, "The pricing of options with default risk," The Journal of Finance, vol. 42, no. 2, pp. 267-280, 1987.

[5] P. Klein, "Pricing black-scholes options with correlated credit risk," Journal of Banking \& Finance, vol. 20, no. 7, pp. 1211-1229, 1996. 
[6] C. H. Hui, C. F. Lo, and H. C. Lee, "Pricing vulnerable BlackScholes options with dynamic default barriers," The Journal of Derivatives, vol. 10, no. 4, pp. 62-69, 2003.

[7] C. H. Hui, C. F. Lo, and K. C. Ku, "Pricing vulnerable European options with stochastic default barriers," IMA Journal of Management Mathematics, vol. 18, no. 4, pp. 315-329, 2007.

[8] P. Klein and M. Inglis, "Valuation of European options subject to financial distress and interest rate risk," The Journal of Derivatives, vol. 6, no. 3, pp. 44-56, 1999.

[9] S.-L. Liao and H.-H. Huang, "Pricing Black-Scholes options with correlated interest rate risk and credit risk: an extension," Quantitative Finance, vol. 5, no. 5, pp. 443-457, 2005.

[10] L. Tian, G. Wang, X. Wang, and Y. Wang, "Pricing vulnerable options with correlated credit risk under jump-diffusion processes," Journal of Futures Markets, vol. 34, no. 10, pp. 957-979, 2013.

[11] H. Niu and D. Wang, "Pricing vulnerable options with correlated jump-diffusion processes depending on various states of the economy," Quantitative Finance, vol. 16, no. 7, pp. 1129-1145, 2016.

[12] M.-K. Lee, S.-J. Yang, and J.-H. Kim, "A closed form solution for vulnerable options with Heston's stochastic volatility," Chaos, Solitons \& Fractals, vol. 86, pp. 23-27, 2016.

[13] G. Wang, X. Wang, and K. Zhou, "Pricing vulnerable options with stochastic volatility," Physica A: Statistical Mechanics and its Applications, vol. 485, pp. 91-103, 2017.

[14] D. S. Bates, "Jumps and stochastic volatility: exchange rate processes implicit in Deutsche Mark options," Review of Financial Studies, vol. 9, no. 1, pp. 69-107, 1996.

[15] P. Carr and L. Wu, "What type of process underlies options? a simple robust test," Journal of Finance, vol. 58, no. 6, pp. 25812610, 2003.

[16] J.-Z. Huang and L. Wu, "Specification analysis of option pricing models based on time-changed Lévy processes," Journal of Finance, vol. 59, no. 3, pp. 1405-1439, 2004.

[17] H. Li, M. T. Wells, and C. L. Yu, "A bayesian analysis of return dynamics with lévy jumps," Review of Financial Studies, vol. 21, no. 5, pp. 2345-2378, 2008.

[18] T. S. Zaevski, Y. S. Kim, and F. J. Fabozzi, “Option pricing under stochastic volatility and tempered stable Lévy jumps," International Review of Financial Analysis, vol. 31, pp. 101-108, 2014.

[19] C. Liang and S. Li, "Option pricing and hedging in incomplete market driven by normal tempered stable process with stochastic volatility," Journal of Mathematical Analysis and Applications, vol. 423, no. 1, pp. 701-719, 2015.

[20] S. Li, Y. Zhou, Y. Wu, and X. Ge, "Equilibrium approach of asset and option pricing under Lévy process and stochastic volatility," Australian Journal of Management, vol. 42, no. 2, pp. 276-295, 2017.

[21] P. Christoffersen, S. Heston, and K. Jacobs, "The shape and term structure of the index option smirk: why multifactor stochastic volatility models work so well," Management Science, vol. 55, no. 12, pp. 1914-1932, 2009.

[22] H. Y. Wong and J. Zhao, "Currency option pricing: Mean reversion and multi-scale stochastic volatility," Journal of Futures Markets, vol. 30, no. 10, pp. 938-956, 2010.

[23] Y. Sun, "Efficient pricing and hedging under the double Heston stochastic volatility jump-diffusion model," International Journal of Computer Mathematics, vol. 92, no. 12, pp. 2551-2574, 2015.
[24] R. C. Merton, "Option pricing when underlying stock returns are discontinuous," Journal of Financial Economics, vol. 3, no. 1-2, pp. 125-144, 1976.

[25] S. G. Kou, "A jump-diffusion model for option pricing," Management Science, vol. 48, no. 8, pp. 1086-1101, 2002.

[26] P. Carr, H. Geman, D. B. Madan, and M. Vor, "The fine structure of asset returns: an empirical investigation," Journal of Business, vol. 75, no. 2, pp. 305-332, 2002.

[27] M. Kendall and A. Stuart, The Advanced Theory of Statistics, vol. 1, Griffin, London, UK, 4th edition, 1977.

[28] N. G. Shephard, "From characteristic function to distribution function: a simple framework for the theory," Econometric Theory, vol. 7, no. 4, pp. 519-529, 1991. 


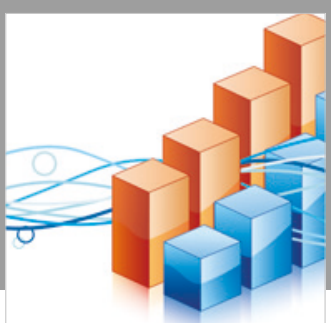

Advances in

Operations Research

\section{-n-m}
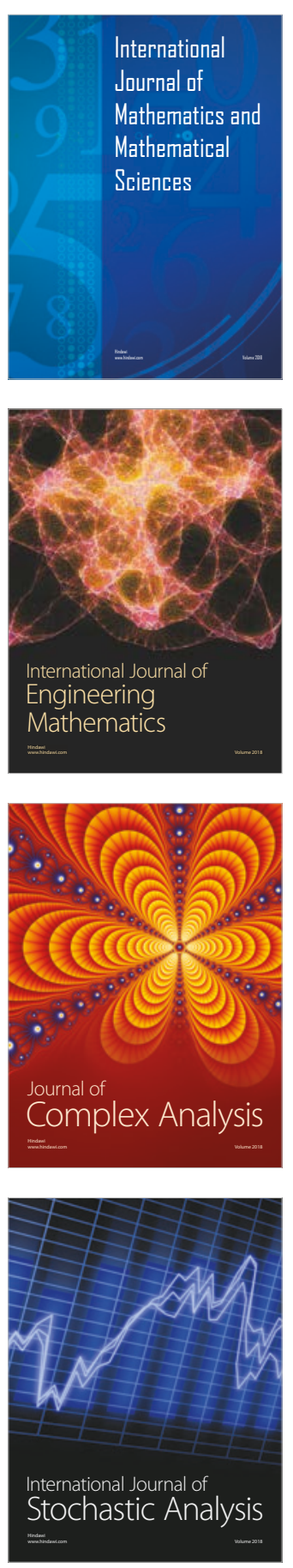
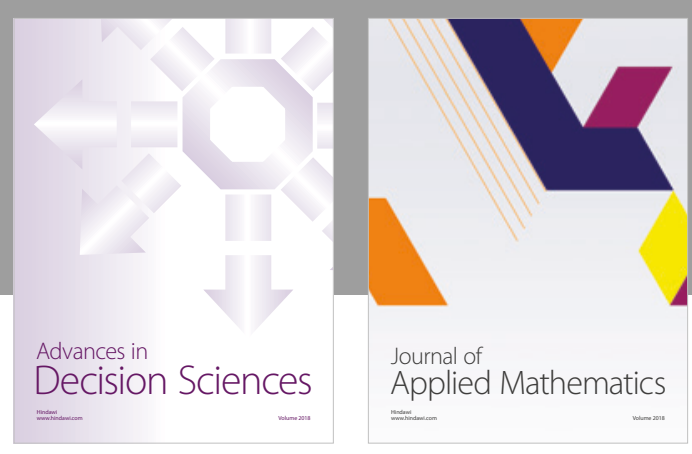

Journal of

Applied Mathematics
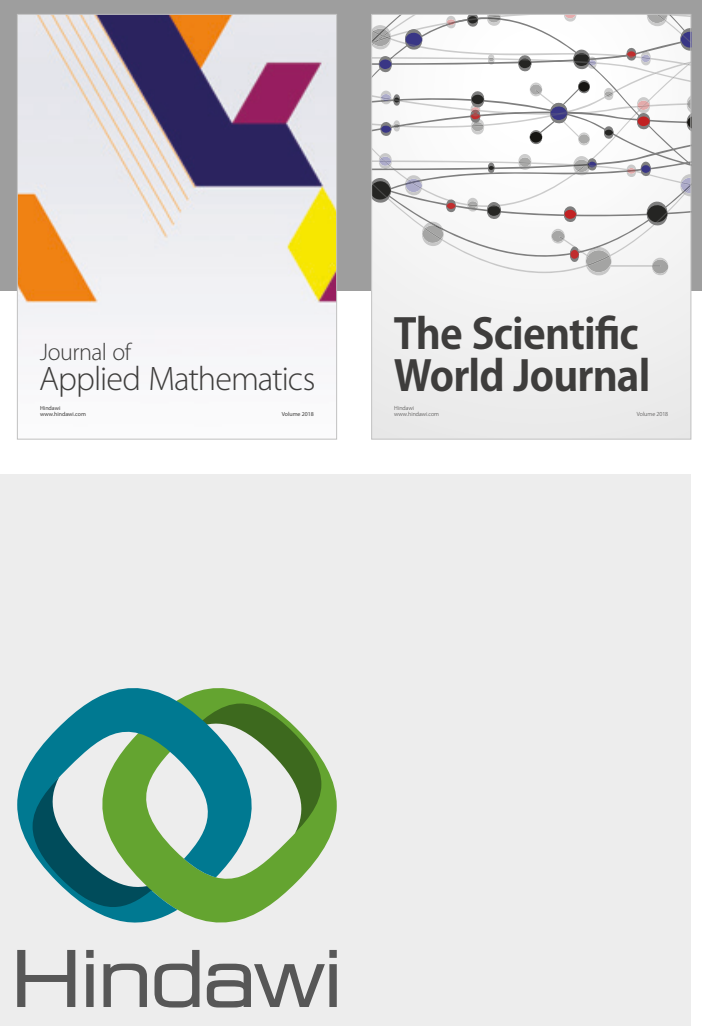

Submit your manuscripts at

www.hindawi.com

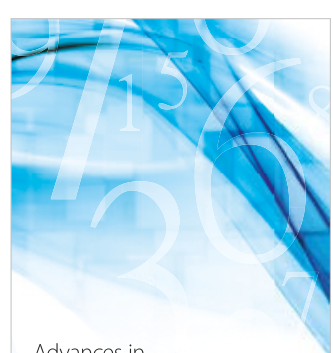

Advances in
Numerical Analysis
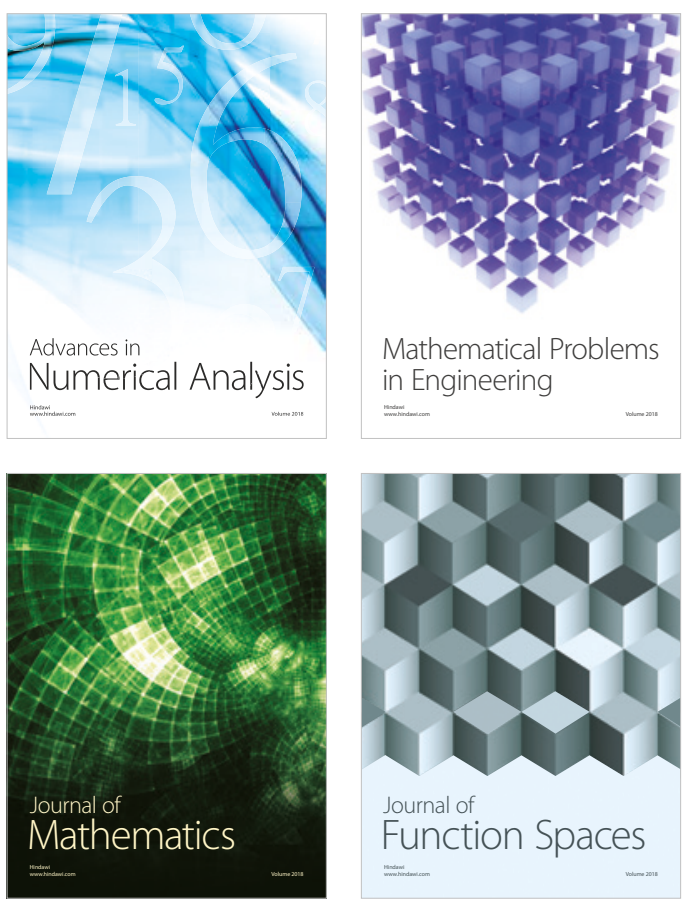

Mathematical Problems in Engineering

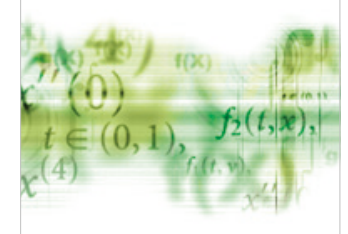

International Journal of

Differential Equations

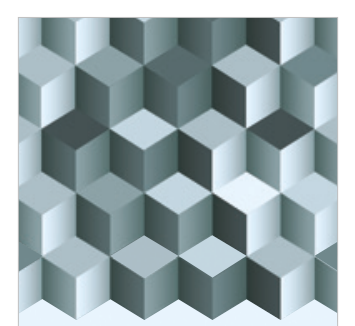

Journal of

Function Spaces

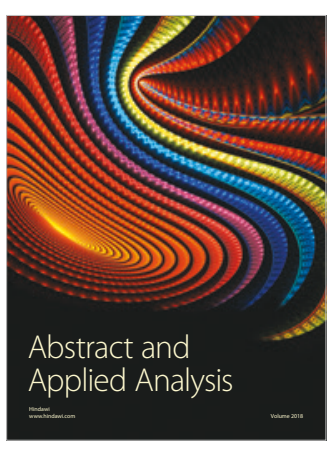

The Scientific

World Journal

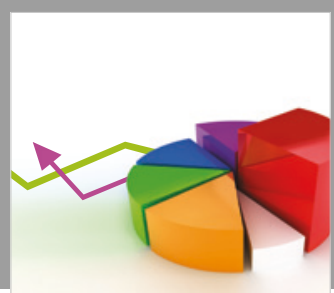

Journal of

Probability and Statistics
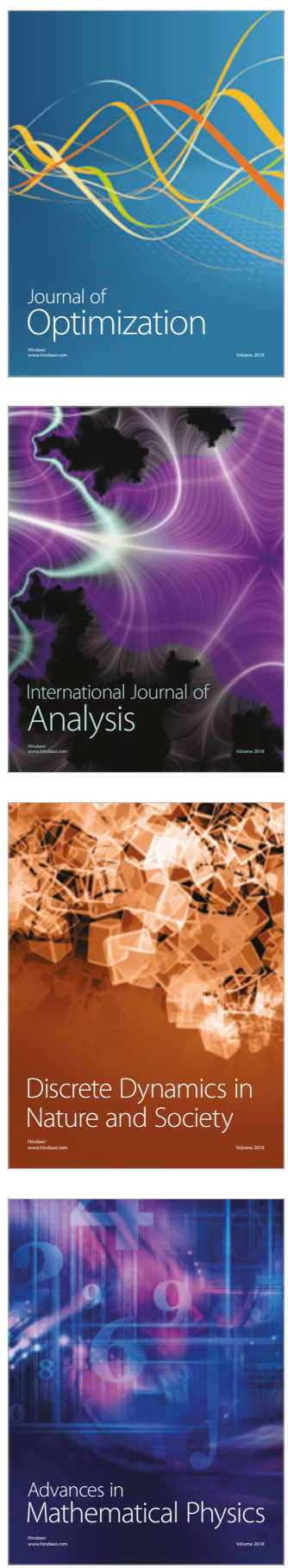